ESAIM: M2AN 55 (2021) 357-380

https://doi.org/10.1051/m2an/2020089
ESAIM: Mathematical Modelling and Numerical Analysis

www.esaim-m2an.org

\title{
VARIOUS CHOICES OF SOURCE TERMS FOR A CLASS OF TWO-FLUID TWO-VELOCITY MODELS
}

\author{
OLIVIER HURISSE*
}

\begin{abstract}
The source terms of the Baer-Nunziato model involve highly non-linear return to equilibrium terms. In order to perform numerical simulations of realistic situations, accounting for this relaxation effects is mandatory. Unfortunately, with the classical forms retained for these source terms in the literature, building efficient, robust and accurate numerical schemes is a tricky task. In this paper, we propose different non-classical forms for these source terms. As for the classical ones, they all agree with the second law of thermodynamics and they are thus associated with a growth of an entropy. The great advantage of some of these new forms of source terms is that they are more linear with respect to the conservative variables. Consequently, this allows to propose more robust, efficient and accurate numerical schemes, in particular when considering fractional step approaches for which source terms and convection terms are solved separately.
\end{abstract}

Mathematics Subject Classification. 76TL10, 35L40.

Received October 14, 2019. Accepted December 21, 2020.

\section{INTRODUCTION}

We consider here the class of the so-called Baer-Nunziato model [1]. In these two-fluid models, each fluid is described by its own pressure, temperature and velocity. The physical coupling of the two fluids is ensured by several ingredients. Firstly, the phasic variables (pressure, temperature and velocity) are supplemented by a fraction. This variable is either a statistical void fraction (see [13,22] for instance) or a volume fraction (see [1] for instance), depending on the modeling processes used to build the model, and it describes the proportion of each phase at a given point in space and at a given time. Secondly, several convective terms involving the fraction appear in the set of partial derivative equations. These terms account for effects that can be seen as interfacial forces due to the space variations of the fraction. They are expressed as non-conservative terms in the momentum equations and in the energy equations of each phase. The modeling of these terms has been widely studied for instance in $[5,7,11,12,14,18,21,22,25]$. Lastly, some source terms are defined in order to account for all the relaxation processes between the phasic quantities: drag force, mass transfer, heat exchange and pressure relaxation. The definition of these source terms relies on the second law of thermodynamics and it thus requires a concave entropy for the mixture of the two-fluids.

Keywords and phrases. Two-fluid two-velocity models, thermodynamical equilibrium, source terms.

EDF Lab Chatou, 6 quai Watier, 78400 Chatou, France.

*Corresponding author: olivier.hurisse@edf.fr 
These two-fluid models have been used for several years in order to perform numerical simulations of unsteady two-phase flows involving heat ant mass transfer $[3,9,16,17,22]$. The numerical schemes used for this kind of simulations are very often based on fractional step approaches [28] that first account for the convective part of the model and then take into account the source terms. Several efficient and robust numerical schemes have been proposed for the numerical discretization of the convective part, see $[4,8,24,26,27]$ among many others. The second step of these approaches then deals with the numerical computation of the source terms, which is associated with complex non-linear ordinary time-derivative equation (ODE) systems. Due to the form of the classical source terms $[1,5,12]$, these ODE systems are highly non-linear, even when dealing with very simple equations of state. Moreover, since the relaxation effects may be very stiff, the numerical schemes have to be both accurate and robust. In the numerical schemes proposed in the literature, the four different relaxation effects (for the velocities, the pressures, the temperatures and the Gibbs free enthalpies) are treated separately $[6,15,16,22,23]$. This strategy has two drawbacks. Firstly, the different effects are numerically decoupled which may lead to predictions with a low accuracy for simulation on coarse meshes. Secondly, the numerical approximation of each relaxation effect is associated with one non-linear ODE system. Hence four non-linear ODE systems have to be solved which is CPU consuming. In the sequel, we therefore propose non-classical forms for these source terms that are more easy to account for in a numerical point of view.

The model and the classical source terms are recalled respectively in Sections 2 and 4 . The non-classical source terms are directly inspired from the thermodynamical source terms that are classically used in two-phase flow homogeneous models, for which it is assumed that both fluids have the same velocity. For the latter, the source terms are often more linear and they can be discretized using very simple and efficient schemes as proposed in $[19,20]$. All the source terms of the sequel are built in order to fulfill the second law of thermodynamics, and several concave entropies are thus defined in Section 3. Four different set of closures for the source terms are proposed in Section 5 on the basis of these different entropies. The first set of closure laws and the second set of closure laws (resp. in Sect. 5.1 and in Sect. 5.2) are presented for the sake of completeness, but they do not seem to be of great interest in a practical point of view for numerical simulations. On the contrary, the third set of closure laws (Sect. 5.3) and the forth set of closure laws (Sect. 5.4) can provide a way to build robust and efficient simulation tools for two-fluid two-velocity models.

\section{A TWO-FLUID TWO-VELOCITY MODEL}

The two-phase flow model considered here belongs to the so-called class of Baer-Nunziato model [1]. Each phase, labeled by an under-script $k=\{l, g\}$, is described by its own specific volume $\tau_{k} \in \mathbb{R}_{*}^{+}$, specific internal energy $e_{k} \in \mathbb{R}_{*}^{+}$and velocity $U_{k} \in \mathbb{R}$; and one Equation of State (EoS) is defined for each fluid in terms of the specific entropy $s_{k}$ :

$$
\left(\tau_{k}, e_{k}\right) \in \mathbb{R}_{*}^{+} \times \mathbb{R}_{*}^{+}:\left(\tau_{k}, e_{k}\right) \mapsto s_{k}\left(\tau_{k}, e_{k}\right) .
$$

We assume the following properties for the specific entropy $s_{k}$.

Definition 2.1. The specific entropy $s_{k}$ :

- belongs to $C^{2}\left(\mathbb{R}_{*}^{+} \times \mathbb{R}_{*}^{+}, \mathbb{R}\right)$;

- is strictly concave with respect to $\left(\tau_{k}, e_{k}\right) \in \mathbb{R}_{*}^{+} \times \mathbb{R}_{*}^{+}$;

- is such that its derivative with respect to the specific internal energy, $\left(s_{k}\right)_{, e_{k}}$, is positive: $\forall\left(\tau_{k}, e_{k}\right) \in \mathbb{R}_{*}^{+} \times$ $\mathbb{R}_{*}^{+},\left(s_{k}\right)_{, e_{k}}>0$.

The following notations are introduced for each phase: the density $\rho_{k}=1 / \tau_{k}$, the total specific energy $E_{k}=e_{k}+U_{k}^{2} / 2$, and $\left(\tau_{k}, e_{k}\right) \mapsto P_{k}\left(\tau_{k}, e_{k}\right)$ the thermodynamical pressure that will be defined more precisely in the following. The void fractions $\alpha_{k} \in \mathbb{R}_{*}^{+}$fulfill the constraint $\alpha_{g}+\alpha_{l}=1$, the partial mass $m_{k}$ of the phase $k$ is denoted by $m_{k}=\alpha_{k} \rho_{k}$, the internal energy per unit of volume $\varepsilon_{k}$ of phase $k$ is denoted by $\varepsilon_{k}=m_{k} e_{k}$, and the total energy per unit of volume $\mathcal{E}_{k}$ is:

$$
\mathcal{E}_{k}=\varepsilon_{k}+\frac{Q_{k}^{2}}{2 m_{k}},
$$


where $Q_{k}=m_{k} U_{k}$ is the momentum of phase $k$ and $U_{k}$ its velocity.

The variable of description of the flow is $X_{g l}=\left(\alpha_{g}, \tau_{g}, e_{g}, U_{g}, \tau_{l}, e_{l}, U_{l}\right)$ and the complete set of equations that rules the time and space evolution of the variables $X_{g l}$ is:

$$
\left\{\begin{array}{l}
\partial_{t}\left(\alpha_{g}\right)+V_{I} \partial_{x}\left(\alpha_{g}\right)=S_{1, g} \\
\partial_{t}\left(\alpha_{g} \rho_{g}\right)+\partial_{x}\left(\alpha_{g} \rho_{g} U_{g}\right)=S_{2, g} \\
\partial_{t}\left(\alpha_{g} \rho_{g} U_{g}\right)+\partial_{x}\left(\alpha_{g} \rho_{g} U_{g}^{2}+\alpha_{g} P_{g}\right)-P_{I} \partial_{x}\left(\alpha_{g}\right)=S_{3, g} \\
\partial_{t}\left(\alpha_{g} \rho_{g} E_{g}\right)+\partial_{x}\left(\alpha_{g} U_{g}\left(\rho_{g} E_{g}+P_{g}\right)\right)+P_{I} \partial_{t}\left(\alpha_{g}\right)=S_{4, g} \\
\partial_{t}\left(\alpha_{l} \rho_{l}\right)+\partial_{x}\left(\alpha_{l} \rho_{l} U_{l}\right)=S_{2, l} \\
\partial_{t}\left(\alpha_{l} \rho_{l} U_{l}\right)+\partial_{x}\left(\alpha_{l} \rho_{l} U_{l}^{2}+\alpha_{l} P_{l}\right)-P_{I} \partial_{x}\left(\alpha_{l}\right)=S_{3, l} \\
\partial_{t}\left(\alpha_{l} \rho_{l} E_{l}\right)+\partial_{x}\left(\alpha_{l} U_{l}\left(\rho_{l} E_{l}+P_{l}\right)\right)+P_{I} \partial_{t}\left(\alpha_{l}\right)=S_{4, l}
\end{array}\right.
$$

In system of equations (2.1) several terms still need to be closed in terms of the variable $X_{g l}$. In this section, we focus on the thermodynamical closure for the pressure $P_{k}$ and of the temperature $T_{k}$; and we recall some classical closure relations for the velocity $V_{I}$ and the pressure term $P_{I}$. The modeling choices for the source terms, $S_{i, k}, i=\{1,2,3,4\}, k=\{l, g\}$, which are the aim of the present work, are discussed in detail in Sections 4 and 5 . We only mention that, for the sake of simplicity, we assume here that they correspond to mass, momentum, and energy exchanges between the two phases. Even if the external exchanges between the two-phase mixture and its surroundings can be taken into account, it is out of the scope of the present work. Hence, for an isolated system, the mass of the mixture $\alpha_{l} \rho_{l}+\alpha_{g} \rho_{g}$, the momentum of the mixture $\alpha_{l} \rho_{l} U_{l}+\alpha_{g} \rho_{g} U_{g}$ and the total energy of the mixture $\alpha_{l} \rho_{l} E_{l}+\alpha_{g} \rho_{g} E_{g}$ must not be modified by these internal exchanges. As a consequence, and adding the constraint $\alpha_{g}+\alpha_{l}=1$, the source terms have to fulfill the relations:

$$
\forall i=\{2,3,4\}, S_{i, l}+S_{i, g}=0 .
$$

Let us now consider regular solutions of system (2.1). The specific entropy $s_{k}$ is a regular function of $\tau_{k}$ and $e_{k}$, see Definition 2.1, so that we have:

$$
\left(\partial_{t}\left(s_{k}\right)+U_{k} \partial_{x}\left(s_{k}\right)\right)=\left(s_{k}\right)_{, \tau_{k}}\left(\partial_{t}\left(\tau_{k}\right)+U_{k} \partial_{x}\left(\tau_{k}\right)\right)+\left(s_{k}\right)_{, e_{k}}\left(\partial_{t}\left(e_{k}\right)+U_{k} \partial_{x}\left(e_{k}\right)\right) .
$$

Then, thanks to the third property of Definition 2.1 for the specific entropy $s_{k}$, we have $\left(s_{k}\right)_{, e_{k}}>0$ and equation (2.3) can be turned to:

$$
\left(\left(s_{k}\right)_{, e_{k}}\right)^{-1} D_{k, t}\left(s_{k}\right)=D_{k, t}\left(e_{k}\right)+\left(\left(s_{k}\right)_{, e_{k}}\right)^{-1}\left(s_{k}\right)_{, \tau_{k}} D_{k, t}\left(\tau_{k}\right),
$$

where the operator $D_{k, t}(\cdot)$ corresponds to the total derivative ${ }^{1}: D_{k, t}(\cdot)=\partial_{t}(\cdot)+U_{k} \partial_{x}(\cdot)$. It is assumed that the classical Gibbs relation holds for each pure phase, that is we have the following relation between the thermodynamical pressure $P_{k}$, the thermodynamical temperature $T_{k}$, and the total derivative of $s_{k}, \tau_{k}$ and $e_{k}$ :

$$
T_{k} D_{k, t}\left(s_{k}\right)=D_{k, t}\left(e_{k}\right)+P_{k} D_{k, t}\left(\tau_{k}\right) .
$$

By identifying the different terms of equations (2.4) and (2.5), we get the thermodynamical definitions of the temperature:

$$
T_{k}=\left(\left(s_{k}\right)_{, e_{k}}\right)^{-1}
$$

and of the pressure

$$
P_{k}=\left(\left(s_{k}\right)_{, e_{k}}\right)^{-1}\left(s_{k}\right)_{, \tau_{k}}=T_{k}\left(s_{k}\right)_{, \tau_{k}}
$$

\footnotetext{
${ }^{1}$ The total derivative gathers the contribution of local derivative of the quantity $\partial_{t}(\cdot)$ and of the convective derivative $U_{k} \partial_{x}(\cdot)$, it corresponds to the derivative along a streamline.
} 
inside each pure phase. The pressure law $\left(\tau_{k}, e_{k}\right) \mapsto P_{k}\left(\tau_{k}, e_{k}\right)$ and the temperature law $\left(\tau_{k}, e_{k}\right) \mapsto T_{k}\left(\tau_{k}, e_{k}\right)$ of each phase are thus directly obtained from the specific entropy $\left(\tau_{k}, e_{k}\right) \mapsto s_{k}\left(\tau_{k}, e_{k}\right)$.

The mass equations (i.e. the second equation and the fifth equation) of (2.1) can be written:

$$
m_{k}\left(\partial_{t}\left(\tau_{k}\right)+U_{k} \partial_{x}\left(\tau_{k}\right)\right)-\alpha_{k} \partial_{x}\left(U_{k}\right)+\left(V_{I}-U_{k}\right) \partial_{x}\left(\alpha_{k}\right)=S_{1, k}-\tau_{k} S_{2, k} .
$$

Furthermore, by combining the momentum equations (i.e. the third equation and the sixth equation) and the mass equations, we obtain from the total energy equations (i.e. the forth equation and the seventh equation) the following equation for the specific internal energy of phase $k$ :

$$
m_{k}\left(\partial_{t}\left(e_{k}\right)+U_{k} \partial_{x}\left(e_{k}\right)\right)+\alpha_{k} P_{k} \partial_{x}\left(U_{k}\right)+P_{I}\left(U_{k}-V_{I}\right) \partial_{x}\left(\alpha_{k}\right)=-P_{I} S_{1, k}-\left(e_{k}+U_{k}^{2} / 2\right) S_{2, k}-U_{k} S_{3, k}+S_{4, k} .
$$

Then, using equations (2.8) and (2.9), one can easily write from (2.3) the following equation for the specific entropy:

$$
\begin{aligned}
m_{k}\left(\partial_{t}\left(s_{k}\right)+U_{k} \partial_{x}\left(s_{k}\right)\right)= & \alpha_{k}\left(\left(s_{k}\right)_{, \tau_{k}}-P_{k}\left(s_{k}\right)_{, e_{k}}\right) \partial_{x}\left(U_{k}\right) \\
& +\left(U_{k}-V_{I}\right)\left(\left(s_{k}\right)_{, \tau_{k}}-P_{I}\left(s_{k}\right)_{, e_{k}}\right) \partial_{x}\left(\alpha_{k}\right) \\
& +\left(\left(s_{k}\right)_{, \tau_{k}}-P_{I}\left(s_{k}\right)_{e_{k}}\right) S_{1, k} \\
& +\left(-\tau_{k}\left(s_{k}\right)_{, \tau_{k}}-\left(e_{k}+U_{k}^{2} / 2\right)\left(s_{k}\right)_{, e_{k}}\right) S_{2, k} \\
& -U_{k}\left(s_{k}\right)_{, e_{k}} S_{3, k} \\
& +\left(s_{k}\right)_{e_{k}} S_{4, k} .
\end{aligned}
$$

Thanks to the definitions of the pressure $P_{k}(2.7)$ and of the temperature $T_{k}$ (2.6), equation (2.10) can be written in conservative form:

$$
\begin{aligned}
\partial_{t}\left(m_{k} s_{k}\right)+\partial_{x}\left(m_{k} U_{k} s_{k}\right)= & \frac{\left(U_{k}-V_{I}\right)\left(P_{k}-P_{I}\right)}{T_{k}} \partial_{x}\left(\alpha_{k}\right) \\
& +\frac{\left(P_{k}-P_{I}\right)}{T_{k}} S_{1, k}+\frac{-\mu_{k}-U_{k}^{2} / 2}{T_{k}} S_{2, k}-\frac{U_{k}}{T_{k}} S_{3, k}+\frac{1}{T_{k}} S_{4, k},
\end{aligned}
$$

where $\mu_{k}=e_{k}+\tau_{k} P_{k}-s_{k} T_{k}$ is the Gibbs enthalpy of phase $k$. This equation for the entropy will be useful in Section 4 in order to define admissible source terms $S_{i, k}$ for the model.

We recall now some classical results for the convective part of the model associated with system of equations (2.1). It can be noted that, as it has been done above for the specific entropy, an equation for the pressure $\left(\tau_{k}, e_{k}\right) \mapsto P_{k}\left(\tau_{k}, e_{k}\right)$ can be obtained by using equations (2.8) and (2.9). When the source terms are omitted, $S_{i, k}=0$, the equation for the pressure reads:

$$
\partial_{t}\left(P_{k}\right)+U_{k} \partial_{x}\left(P_{k}\right)+\rho_{k} C_{k}^{2} \partial_{x}\left(U_{k}\right)-\frac{\left(U_{k}-V_{I}\right)}{m_{k}}\left(\left(P_{k}\right)_{\tau_{k}}-P_{I}\left(P_{k}\right)_{, e_{k}}\right) \partial_{x}\left(\alpha_{k}\right)=0,
$$

where the sound speed $\left(\tau_{k}, e_{k}\right) \mapsto C_{k}\left(\tau_{k}, e_{k}\right)$ for phase $k$ has been introduced:

$$
C_{k}^{2}=\tau_{k}^{2}\left(P_{k}\left(P_{k}\right)_{e_{k}}-\left(P_{k}\right)_{\tau_{k}}\right) \text {. }
$$

Thanks to the definitions of the pressure $P_{k}(2.7)$ and of the temperature $T_{k}(2.6)$, relation (2.13) can also be written:

$$
\frac{C_{k}^{2}}{T_{k} \tau_{k}^{2}}=-\left(-1, P_{k}\right) \cdot s_{k}^{\prime \prime} \cdot\left(\begin{array}{c}
-1 \\
P_{k}
\end{array}\right)
$$

In relation (2.14), the matrix $s_{k}^{\prime \prime}$ stands for the Hessian matrix of the phasic entropy $\left(\tau_{k}, e_{k}\right) \mapsto s_{k}\left(\tau_{k}, e_{k}\right)$, which is defined since $s_{k}$ belongs to $C^{2}\left(\mathbb{R}_{*}^{+} \times \mathbb{R}_{*}^{+}\right)$(see Def. 2.1). The following proposition then holds. 
Proposition 2.2. Under the assumptions of Definition 2.1, we have: $\forall\left(\tau_{k}, e_{k}\right) \in \mathbb{R}_{*}^{+} \times \mathbb{R}_{*}^{+}, C_{k}\left(\tau_{k}, e_{k}\right) \in \mathbb{R}_{*}^{+}$.

Proof. Thanks to the properties of the specific entropy $s_{k}$ (see Def. 2.1), $T_{k}>0$ and $-s_{k}^{\prime \prime}$ is symmetric definite strictly positive. Hence, equation (2.14) gives $C_{k}^{2}>0$ for all $\left(\tau_{k}, e_{k}\right) \in \mathbb{R}_{*}^{+} \times \mathbb{R}_{*}^{+}$, and thus $C_{k}$ is a positive real quantity.

Let us introduce now the classical choice proposed in [11] for the velocity $V_{I}$ which reads:

$$
V_{I}=\beta U_{l}+(1-\beta) U_{g},
$$

and for the pressure $P_{I}$ :

$$
P_{I}=\frac{(1-\beta)\left(\left(s_{l}\right)_{, \tau_{l}}\right)+\beta\left(\left(s_{g}\right)_{,_{g}}\right)}{(1-\beta)\left(\left(s_{l}\right)_{,_{l}}\right)+\beta\left(\left(s_{g}\right)_{e_{g}}\right)}=\frac{(1-\beta) P_{l} / T_{l}+\beta P_{g} / T_{g}}{(1-\beta) / T_{l}+\beta / T_{g}},
$$

where the parameter $\beta$ has three possible forms:

- $\beta=0$ or $\beta=1$, which corresponds to the classical Baer-Nunziato model [1];

- or $\beta=m_{l} /\left(m_{l}+m_{g}\right)$.

These different choices for $\beta$ have been studied with the help of numerical simulations in [14,23].

With the help of the closure laws (2.6), (2.7), (2.15), (2.16), the convective part of system (2.1) (i.e. with $\left.S_{i, k}=0\right)$ is closed and several properties can be exhibited.

Proposition 2.3. With the velocity $V_{I}$ defined by (2.15) and the pressure $P_{I}$ defined by (2.16):

- (hyperbolicity) system (2.1) possesses seven real eigenvalues $V_{I}, U_{k}, U_{k}-C_{k}, k=\{1,2\}$ and the associated eigenvectors form a basis of $\mathbb{R}^{7}$, provided that resonance does not occur:

$$
\left(U_{k}-V_{I}\right)^{2} \neq C_{k}^{2}, k=\{1,2\}
$$

- the field $\alpha_{g}$ is associated with the eigenvalue $V_{I}$ which is a linearly degenerate field;

- system (2.1) admits a symmetric form.

Proof. The proof of the items of Proposition 2.3 is based on the study of the eigenstructure of the convective part of system (2.1). For the first item and the second item the detailed proof can be found for instance in [11]. The third item has been shown in [7].

Remark 2.4. It should be noted that $\alpha_{k}=0$ or $m_{k}=0$ corresponds to single phase situations that can not be handled properly through the set of PDE's (2.1). Indeed, for the single phase situations system of equations (2.1) does not allow to define uniquely all the quantities. We thus consider that these situations are out of the scope of the two-phase flow model considered here.

The convective part has been closed thanks to relations $(2.6),(2.7),(2.15),(2.16)$. The remaining of the paper is dedicated to the closure relations for the source terms $S_{i, k}, i=\{1,2,3,4\}, k=\{l, g\}$. For that purpose, we first define in Section 3 several concave entropies for the mixture. These entropies will be used in Sections 4 and 5 in order to define sources terms that ensure an entropy inequality.

\section{Definition of some Entropies}

In order to propose some closure laws for the source terms $S_{i, k}, i=\{1,2,3,4\}, k=\{l, g\}$, we proceed here following a classical approach, see $[6,7,11,12,22]$ among many other references: admissible forms for the source terms must agree with the second law of thermodynamics associated with a concave mixture entropy. For that purpose, several entropies can be considered and in this section some of them are investigated. In Section 3.1 we focus on mixture entropies that only account for the thermodynamical aspects of the model, that is they only depend on the thermodynamical quantities and not on the momentums. Whereas in Section 3.2, entropies for the whole model are considered. 


\subsection{Definition of thermodynamical entropies for the mixture}

Let us define the thermodynamical mixture entropy $\eta$ as the weighted average of the phasic specific entropy:

$$
\eta:\left(\begin{array}{l}
H_{\eta} \rightarrow \mathbb{R} \\
\left.Z_{g l} \mapsto m_{g} s_{g}\left(\frac{\alpha_{g}}{m_{g}}, \frac{\varepsilon_{g}}{m_{g}}\right)+m_{l} s_{l}\left(\frac{\alpha_{l}}{m_{l}}, \frac{\varepsilon_{l}}{m_{l}}\right)\right),
\end{array}\right.
$$

where $Z_{g l}=\left(\alpha_{g}, m_{g}, \varepsilon_{g}, \alpha_{l}, m_{l}, \varepsilon_{l}\right)$ is a vector of variables belonging to the set: $H_{\eta}=\left(\mathbb{R}_{*}^{+} \times \mathbb{R}_{*}^{+} \times \mathbb{R}_{*}^{+}\right)^{2}$. It should be noticed that the constraint $\left(\alpha_{g}+\alpha_{l}\right)=1$ on the fraction is not yet accounted for in definition of the entropy $\eta$ (3.1). It will be introduced latter. The entropy $\eta$ inherits from the phasic specific entropies $s_{k}$ several properties.

Proposition 3.1. Under the assumptions of Definition 2.1, the mixture entropy $Z_{g l} \mapsto \eta\left(Z_{g l}\right)$ has the following properties:

- $\forall a \in \mathbb{R}^{+}, \forall Z \in H_{\eta}, \eta(a Z)=a \eta(Z) ;$

- $\eta$ belongs to $C^{2}\left(H_{\eta}, \mathbb{R}\right)$;

- $Z_{g l} \mapsto \eta\left(Z_{g l}\right)$ is concave on $H_{\eta}$, its degeneracy manifold is:

$$
\mathcal{M}_{\eta}\left(Z_{g l}\right)=\left\{a\left(\alpha_{g}, m_{g}, \varepsilon_{g}, 0,0,0\right)+b\left(0,0,0, \alpha_{l}, m_{l}, \varepsilon_{l}\right),(a, b) \in \mathbb{R}^{2}\right\}
$$

Proof. The first item of Proposition 3.1 is obvious, it simply arises from the definition of $\eta$. In order to prove the second item and the third item, let us introduce two vectors that gather the phasic quantity of $Z_{g l}: Z_{k}=$ $\left(\alpha_{k}, m_{k}, \varepsilon_{k}\right)$; and the two phasic entropies $\eta_{k}$ :

$$
\eta_{k}:\left(\begin{array}{l}
H_{\eta, k} \rightarrow \mathbb{R} \\
Z_{k} \mapsto m_{k} s_{k}\left(\frac{\alpha_{k}}{m_{k}}, \frac{\varepsilon_{k}}{m_{k}}\right)
\end{array}\right),
$$

where $H_{\eta, k}=\mathbb{R}_{*}^{+} \times \mathbb{R}_{*}^{+} \times \mathbb{R}_{*}^{+}$. It follows from these definitions that the mixture entropy can be written using a separation of variables:

$$
\eta:\left(\begin{array}{l}
H_{\eta, g} \times H_{\eta, l} \rightarrow \mathbb{R} \\
\left(Z_{g}, Z_{l}\right) \mapsto \eta_{g}\left(Z_{g}\right)+\eta_{l}\left(Z_{l}\right)
\end{array}\right) .
$$

As a consequence, since the phasic entropy $\left(\tau_{k}, e_{k}\right) \mapsto s_{k}\left(\tau_{k}, e_{k}\right)$ belongs to $C^{2}\left(\mathbb{R}_{*}^{+} \times \mathbb{R}_{*}^{+}, \mathbb{R}\right)$ and since $m_{k}>0$, the phasic entropy $Z_{k} \mapsto \eta_{k}\left(Z_{k}\right)$ defined by (3.3) belongs to $C^{2}\left(H_{\eta, k}, \mathbb{R}\right)$. Thanks to the separation of variables (3.4) for $\eta$, one easily obtains the second item of Proposition 3.1. In order to prove the concavity of $\eta$, we compute the Hessian matrix for each phasic entropy $\eta_{k}$. We first begin by computing the first derivatives of $\eta_{k}$ :

$$
\begin{aligned}
& \partial_{\alpha_{k}}\left(\eta_{k}\right)_{\mid m_{k}, \varepsilon_{k}}\left(Z_{k}\right)=\partial_{\tau_{k}}\left(s_{k}\right)\left(\frac{\alpha_{k}}{m_{k}}, \frac{\varepsilon_{k}}{m_{k}}\right) \\
& \partial_{m_{k}}\left(\eta_{k}\right)_{\mid \alpha_{k}, \varepsilon_{k}}\left(Z_{k}\right)=s_{k}\left(\frac{\alpha_{k}}{m_{k}}, \frac{\varepsilon_{k}}{m_{k}}\right)-\frac{\alpha_{k}}{m_{k}} \partial_{\tau_{k}}\left(s_{k}\right)\left(\frac{\alpha_{k}}{m_{k}}, \frac{\varepsilon_{k}}{m_{k}}\right)-\frac{\varepsilon_{k}}{m_{k}} \partial_{e_{k}}\left(s_{k}\right)\left(\frac{\alpha_{k}}{m_{k}}, \frac{\varepsilon_{k}}{m_{k}}\right) \\
& \partial_{\varepsilon_{k}}\left(\eta_{k}\right)_{\mid \alpha_{k}, m_{k}}\left(Z_{k}\right)=\partial_{e_{k}}\left(s_{k}\right)\left(\frac{\alpha_{k}}{m_{k}}, \frac{\varepsilon_{k}}{m_{k}}\right) .
\end{aligned}
$$


Then the second derivatives are computed and we find:

$$
\begin{aligned}
\partial_{\alpha_{k}, \alpha_{k}}^{2}\left(\eta_{k}\right) & =\frac{1}{m_{k}} \partial_{\tau_{k}, \tau_{k}}^{2}\left(s_{k}\right) \\
\partial_{\alpha_{k}, m_{k}}^{2}\left(\eta_{k}\right) & =-\frac{\alpha_{k}}{m_{k}^{2}} \partial_{\tau_{k}, \tau_{k}}^{2}\left(s_{k}\right)-\frac{\varepsilon_{k}}{m_{k}^{2}} \partial_{\tau_{k}, e_{k}}^{2}\left(s_{k}\right) \\
\partial_{\alpha_{k}, \varepsilon_{k}}^{2}\left(\eta_{k}\right) & =\frac{1}{m_{k}} \partial_{\tau_{k}, e_{k}}^{2}\left(s_{k}\right) \\
\partial_{\varepsilon_{k}, \varepsilon_{k}}^{2}\left(\eta_{k}\right) & =\frac{1}{m_{k}} \partial_{e_{k}, e_{k}}^{2}\left(s_{k}\right) \\
\partial_{m_{k}, \varepsilon_{k}}^{2}\left(\eta_{k}\right) & =-\frac{\alpha_{k}}{m_{k}^{2}} \partial_{\tau_{k}, e_{k}}^{2}\left(s_{k}\right)-\frac{\varepsilon_{k}}{m_{k}^{2}} \partial_{e_{k}, e_{k}}^{2}\left(s_{k}\right) \\
\partial_{m_{k}, m_{k}}^{2}\left(\eta_{k}\right) & =\frac{1}{m_{k}}\left(\frac{\alpha_{k}^{2}}{m_{k}^{2}}, \frac{\varepsilon_{k}^{2}}{m_{k}^{2}}\right) \cdot s_{k}^{\prime \prime} \cdot\left(\begin{array}{l}
\frac{\alpha_{k}^{2}}{m_{k}^{2}} \\
\frac{\varepsilon_{k}^{2}}{m_{k}^{2}}
\end{array}\right)
\end{aligned}
$$

In the second derivative term (3.13), $s_{k}^{\prime \prime}$ stands for the Hessian matrix of the phasic entropy $\left(\tau_{k}, e_{k}\right) \mapsto s_{k}\left(\tau_{k}, e_{k}\right)$. It is worth noting that thanks to the strict concavity of $s_{k}$ on $H_{\eta, k}, \partial_{m_{k}, m_{k}}^{2}\left(\eta_{k}\right)<0$ on $H_{\eta, k}$. As a consequence, the function:

$$
\left(\begin{array}{l}
\mathbb{R}_{*}^{+} \rightarrow \mathbb{R} \\
m_{k} \mapsto \eta_{k}\left(\alpha_{k}, m_{k}, \varepsilon_{k}\right)
\end{array}\right)
$$

is strictly concave on $\mathbb{R}_{*}^{+}$. With the second derivatives (3.8)-(3.13), the Hessian matrix $\eta_{k}^{\prime \prime}$ of $Z_{k} \mapsto \eta_{k}\left(Z_{k}\right)$ can be explicitly written. In particular, we have for any vector $\left(x_{k}, y_{k}, z_{k}\right) \in \mathbb{R}^{3}$ :

$$
\left(x_{k}, y_{k}, z_{k}\right) \cdot \eta_{k}^{\prime \prime} \cdot\left(\begin{array}{l}
x_{k} \\
y_{k} \\
z_{k}
\end{array}\right)=\left(x_{k}-y_{k} \frac{\alpha_{k}}{m_{k}}, z_{k}-y_{k} \frac{\varepsilon_{k}}{m_{k}}\right) \cdot s_{k}^{\prime \prime} \cdot\left(\begin{array}{c}
x_{k}-y_{k} \frac{\alpha_{k}}{m_{k}} \\
z_{k}-y_{k} \frac{\varepsilon_{k}}{m_{k}}
\end{array}\right) .
$$

Then thanks to the variable separation (3.4) for $\eta$, one can obtain from the Hessian matrix $\eta^{\prime \prime}$ of $Z_{g l} \mapsto \eta\left(Z_{g l}\right)$ and for any $\mathcal{X}=\left(x_{g}, y_{g}, z_{g}, x_{l}, y_{l}, z_{l}\right) \in \mathbb{R}^{6}$ :

$$
\mathcal{X} \cdot \eta^{\prime \prime} \cdot \mathcal{X}^{\top}=\sum_{k}\left(x_{k}-y_{k} \frac{\alpha_{k}}{m_{k}}, z_{k}-y_{k} \frac{\varepsilon_{k}}{m_{k}}\right) \cdot s_{k}^{\prime \prime} \cdot\left(\begin{array}{c}
x_{k}-y_{k} \frac{\alpha_{k}}{m_{k}} \\
z_{k}-y_{k} \frac{\varepsilon_{k}}{m_{k}}
\end{array}\right)
$$

Since the phasic entropies $s_{k}$ are concave, we can conclude that

$$
\mathcal{X} \cdot \eta^{\prime \prime} \cdot \mathcal{X}^{\top} \leq 0
$$

and hence that $Z_{g l} \mapsto \eta\left(Z_{g l}\right)$ is concave but not strictly concave. Indeed, the degeneracy manifold $\mathcal{M}_{\eta}\left(Z_{g l}\right)$ of entropy $\eta$ at a point $Z_{g l} \in H_{\eta}$ can be found as the set of vectors that are such that

$$
\mathcal{X} \cdot \eta^{\prime \prime} \cdot \mathcal{X}^{\top}=0
$$

Due to the strict concavity of $s_{k}$, one can obtain from relation (3.16):

$$
\mathcal{M}_{\eta}\left(Z_{g l}\right)=\left\{a\left(\alpha_{g}, m_{g}, \varepsilon_{g}, 0,0,0\right)+b\left(0,0,0, \alpha_{l}, m_{l}, \varepsilon_{l}\right),(a, b) \in \mathbb{R}^{2}\right\} .
$$

Since $\alpha_{k}>0, m_{k}>0$ and $\varepsilon_{k}>0$, the degeneracy manifold $\mathcal{M}_{\eta}\left(Z_{g l}\right)$ is a vector subspace of dimension 2 of $\mathbb{R}^{6}$. This ends the proof of Proposition 3.1.

From the proof of Proposition 3.1, one can get an interesting auxillary result for the entropy $m_{k} \mapsto \eta_{m, k}\left(m_{k}\right)$ defined by (3.14). This result will be used in Section 5.4. The properties of the entropy $s_{k}$ lead to the following proposition. 
Proposition 3.2. The function $m_{k} \mapsto \eta_{m, k}\left(m_{k}\right)$ defined for given $\alpha_{k} \in \mathbb{R}_{*}^{+}$and $\varepsilon_{k} \in \mathbb{R}_{*}^{+}$by:

$$
\eta_{m, k}:\left(\begin{array}{l}
\mathbb{R}_{*}^{+} \rightarrow \mathbb{R} \\
m_{k} \mapsto \eta_{k}\left(\alpha_{k}, m_{k}, \varepsilon_{k}\right)
\end{array}\right)
$$

belongs to $C^{2}\left(\mathbb{R}_{*}^{+}, \mathbb{R}\right)$ and it is strictly concave on $\mathbb{R}_{*}^{+}$.

Proof. Since the entropy $\eta_{k}$ belongs to $C^{2}\left(\mathbb{R}_{*}^{+} \times \mathbb{R}_{*}^{+}, \mathbb{R}\right), m_{k} \mapsto \eta_{m, k}\left(m_{k}\right)$ obviously belongs to $C^{2}\left(\mathbb{R}_{*}^{+}, \mathbb{R}\right)$. Thanks to equation (3.13), the second derivative of $m_{k} \mapsto \eta_{m, k}\left(m_{k}\right)$ reads:

$$
\eta_{m, k}^{\prime \prime}\left(m_{k}\right)=\frac{1}{m_{k}}\left(\frac{\alpha_{k}^{2}}{m_{k}^{2}}, \frac{\varepsilon_{k}^{2}}{m_{k}^{2}}\right) \cdot s_{k}^{\prime \prime} \cdot\left(\begin{array}{c}
\frac{\alpha_{k}^{2}}{m_{k}^{2}} \\
\frac{\varepsilon_{k}^{2}}{m_{k}^{2}}
\end{array}\right) .
$$

Since the phasic entropies $s_{k}$ are strictly concave, we can conclude that:

$$
\forall m_{k} \in \mathbb{R}_{*}^{+}, \eta_{m, k}^{\prime \prime}\left(m_{k}\right)<0
$$

This ends the proof of Proposition 3.2.

We define now the entropy $\widetilde{\eta}$ that is the restriction of $\eta$ on $\widetilde{H}_{\eta, 0}$, a subset of $H_{\eta}$ :

$$
\widetilde{\eta}:\left(\begin{array}{l}
\widetilde{H}_{\eta, 0} \rightarrow \mathbb{R} \\
Z_{g l} \mapsto \eta\left(Z_{g l}\right)
\end{array}\right)
$$

The domain $\widetilde{H}_{\eta, 0}$ is the subset of $H_{\eta}$ that corresponds to a given sum for the fractions $\alpha_{g}+\alpha_{l}=\alpha_{0}$, for the partial masses $m_{g}+m_{l}=m_{0}$ and for the total energies $\mathcal{E}_{g}+\mathcal{E}_{l}=\mathcal{E}_{0}$ :

$$
\widetilde{H}_{\eta, 0}=\left\{Z_{g l} \in H_{\eta} ; \alpha_{g}+\alpha_{l}=\alpha_{0}, m_{g}+m_{l}=m_{0}, \mathcal{E}_{g}+\mathcal{E}_{l}=\mathcal{E}_{0}\right\} .
$$

It can easily be shown that this domain $\widetilde{H}_{\eta, 0}$ is a bounded convex subset of $\left(\mathbb{R}^{+}\right)^{6}$. With this restriction of the domain of definition, the entropy $\widetilde{\eta}$ has the following property.

Theorem 3.3. The entropy $Z_{g l} \mapsto \widetilde{\eta}\left(Z_{g l}\right)$ defined by (3.20) and (3.21) is strictly concave on $\widetilde{H}_{\eta, 0}$, except at the points $Z_{g l}$ for which there exists $\kappa \in \mathbb{R}_{*}^{+}$such that:

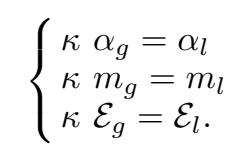

In such situations, the degeneracy manifold of $Z_{g l} \mapsto \widetilde{\eta}\left(Z_{g l}\right)$ is the sub-space of $\mathbb{R}^{6}$ :

$$
\mathcal{M}_{\eta}\left(Z_{g l}\right) \cap \widetilde{H}_{\eta, 0}=\left\{\left(\alpha_{0}, m_{0}, \mathcal{E}_{0}, 0,0,0\right)+b\left(-\alpha_{l},-m_{l},-\mathcal{E}_{l}, \alpha_{l}, m_{l}, \mathcal{E}_{l}\right), b \in \mathbb{R}\right\} .
$$

Proof. From Proposition 3.1, we can deduce that $Z_{g l} \mapsto \widetilde{\eta}\left(Z_{g l}\right)$ is concave on $\widetilde{H}_{\eta, 0} \subset H_{\eta}$. In order to exhibit the form (3.22) of the manifold, let us define a point which belongs to the degeneracy manifold of $\widetilde{\eta}$ at point $Z_{g l}$ defined by $(3.2)$ and to $\widetilde{H}_{\eta, 0}$ :

$$
Z_{g l}=\left(\alpha_{g}, m_{g}, \mathcal{E}_{g}, \alpha_{l}, m_{l}, \mathcal{E}_{l}\right) \in \mathcal{M}_{\eta}\left(Z_{g l}\right) \cap \widetilde{H}_{\eta, 0} .
$$

By definition of $\mathcal{M}_{\eta}\left(Z_{g l}\right)$ and $\widetilde{H}_{\eta, 0}$, there exist $(a, b) \in \mathbb{R}^{2}$ such that:

$$
\left\{\begin{array}{l}
\alpha_{g}+\alpha_{l}=a \alpha_{g}+b \alpha_{l}=\alpha_{0} \\
m_{g}+m_{l}=a m_{g}+b m_{l}=m_{0} \\
\mathcal{E}_{g}+\mathcal{E}_{l}=a \mathcal{E}_{g}+b \mathcal{E}_{l}=\mathcal{E}_{0}
\end{array}\right.
$$


These equations lead to the system:

$$
\left\{\begin{array}{l}
(a-1) \alpha_{g}+(b-1) \alpha_{l}=0 \\
(a-1) m_{g}+(b-1) m_{l}=0 \\
(a-1) \mathcal{E}_{g}+(b-1) \mathcal{E}_{l}=0 .
\end{array}\right.
$$

If at least two among the three equations of system (3.23) are linearly independent, there exists a unique solution $(a, b)$ to system (3.23): $a=b=1$. Hence, in such a situation, the intersection of the degeneracy manifold $\mathcal{M}_{\eta}\left(Z_{g l}\right)$ with the domain $\widetilde{H}_{\eta, 0}$ is restricted to a single point:

$$
\mathcal{M}_{\eta}\left(Z_{g l}\right) \cap \widetilde{H}_{\eta, 0}=\left\{Z_{g l}\right\} .
$$

The entropy $Z_{g l} \mapsto \widetilde{\eta}\left(Z_{g l}\right)$ is then strictly concave. On the contrary, when the three equations of (3.23) are equivalent, the solution $(a, b)$ is not unique. This situation occurs when there exists $\kappa \in \mathbb{R}_{*}^{+}$such that:

$$
\left\{\begin{array}{l}
\kappa \alpha_{g}=\alpha_{l} \\
\kappa m_{g}=m_{l} \\
\kappa \mathcal{E}_{g}=\mathcal{E}_{l}
\end{array}\right.
$$

and system (3.23) leads to the relation:

$$
a=1+(1-b) \kappa .
$$

In such a situation, the entropy $Z_{g l} \mapsto \widetilde{\eta}\left(Z_{g l}\right)$ is not strictly concave and its degeneracy manifold is the sub-space of $\mathbb{R}^{6}$ :

$$
\mathcal{M}_{\eta}\left(Z_{g l}\right) \cap \widetilde{H}_{\eta, 0}=\left\{\left(\alpha_{0}, m_{0}, \mathcal{E}_{0}, 0,0,0\right)+b\left(-\alpha_{l},-m_{l},-\mathcal{E}_{l}, \alpha_{l}, m_{l}, \mathcal{E}_{l}\right), b \in \mathbb{R}_{*}^{+}\right\} .
$$

This ends the proof of Theorem 3.3.

The entropy $Z_{g l} \mapsto \widetilde{\eta}\left(Z_{g l}\right)$ is thus not strictly concave. Nonetheless, under additional assumptions on the phasic entropies $s_{k}$, a stronger result can be stated. This result is given formally in Corollary 3.4. With Theorem 3.3, it represents a key point in the non-classical formulation of the source terms of Section 5.

Corollary 3.4. If we assume that the two phasic entropies $\left(\tau_{k}, e_{k}\right) \mapsto s_{k}\left(\tau_{k}, e_{k}\right)$ are such that for all $(\tau, e) \in$ $\mathbb{R}_{*}^{+} \times \mathbb{R}_{*}^{+}$, we have:

$$
s_{l}(\tau, e) \neq s_{g}(\tau, e) \quad \text { or } \quad \partial_{\tau_{l}}\left(s_{l}\right)_{\mid e_{l}}(\tau, e) \neq \partial_{\tau_{g}}\left(s_{g}\right)_{\mid e_{g}}(\tau, e) \quad \text { or } \quad \partial_{e_{l}}\left(s_{l}\right)_{\mid \tau_{l}}(\tau, e) \neq \partial_{e_{g}}\left(s_{g}\right)_{\mid \tau_{g}}(\tau, e),
$$

then the mixture entropy $Z_{g l} \mapsto \widetilde{\eta}\left(Z_{g l}\right)$ admits a unique maximum on its domain of definition $\widetilde{H}_{\eta, 0}$.

Proof. In order to prove Corollary 3.4, the results of Theorem 3.3 and some elements of its proof are used. From the latter, we know that $\widetilde{\eta}$ is concave on the bounded convex set $\widetilde{H}_{\eta, 0}$. But $\widetilde{\eta}$ is not strictly concave and its maximum may a priori be reached for several points in $\widetilde{H}_{\eta, 0}$. Since $\widetilde{\eta}$ belongs to $C^{2}\left(\widetilde{H}_{\eta}, \mathbb{R}\right)$ (see Prop. 3.1), the set of these points,

$$
\Theta_{\widetilde{\eta}}=\left\{Z_{g l}^{\prime} \in \widetilde{H}_{\eta, 0} ; \forall Z_{g l} \in \widetilde{H}_{\eta, 0}, \widetilde{\eta}\left(Z_{g l}^{\prime}\right) \geq \widetilde{\eta}\left(Z_{g l}\right)\right\},
$$

is an open bounded convex subset of $\widetilde{H}_{\eta, 0}$ (which is also an open bounded convex set). From (3.20) and (3.21), we know that the entropy $\widetilde{\eta}$ is defined as the restriction of the entropy $\eta$ to the domain $H_{\eta}$ with the three linear constraints:

$$
\left\{\begin{array}{l}
G_{1}\left(Z_{g l}\right)=\alpha_{g}+\alpha_{l}-1=0 \\
G_{2}\left(Z_{g l}\right)=m_{g}+m_{l}-m_{0}=0 \\
G_{3}\left(Z_{g l}\right)=\mathcal{E}_{g}+\mathcal{E}_{l}-\mathcal{E}_{0}=0
\end{array}\right.
$$


whose gradients with respect to $Z_{g l}$ are independent and read:

$$
\left\{\begin{array}{l}
\nabla_{Z_{g l}} G_{1}=(1,0,0,1,0,0)^{\top} \\
\nabla_{Z_{g l}} G_{2}=(0,1,0,0,1,0)^{\top} \\
\nabla_{Z_{g l}} G_{3}=(0,0,1,0,0,1)^{\top}
\end{array}\right.
$$

Therefore, there exists three Lagrange multipliers $a_{1}, a_{2}$ and $a_{3}$ such that for a maximizer $Z_{g l}$ of $\widetilde{\eta}$ we have:

$$
\nabla_{Z_{g l}} \widetilde{\eta}\left(Z_{g l}\right)=a_{1} \nabla_{Z_{g l}} G_{1}\left(Z_{g l}\right)+a_{2} \nabla_{Z_{g l}} G_{2}\left(Z_{g l}\right)+a_{3} \nabla_{Z_{g l}} G_{3}\left(Z_{g l}\right),
$$

and thus the first order conditions for the existence of the maximum $Z_{g l}$ are:

$$
\left\{\begin{array}{l}
\partial_{\alpha_{g}}(\widetilde{\eta})\left(Z_{g l}\right)=a_{1}=\partial_{\alpha_{l}}(\widetilde{\eta})\left(Z_{g l}\right) \\
\partial_{m_{g}}(\widetilde{\eta})\left(Z_{g l}\right)=a_{2}=\partial_{m_{l}}(\widetilde{\eta})\left(Z_{g l}\right) \\
\partial_{\mathcal{E}_{g}}(\widetilde{\eta})\left(Z_{g l}\right)=a_{3}=\partial_{\mathcal{E}}(\widetilde{\eta})\left(Z_{g l}\right)
\end{array}\right.
$$

Relations (3.28) can also be written in terms of the phasic entropies $s_{k}$, the phasic specific volumes $\tau_{k}$ and the phasic specific energies $e_{k}$ :

$$
\left\{\begin{array}{l}
\partial_{\tau_{l}}\left(s_{l}\right)_{\mid e_{l}}\left(\tau_{l}, e_{l}\right)=\partial_{\tau_{g}}\left(s_{g}\right)_{\mid e_{g}}\left(\tau_{g}, e_{g}\right), \\
\partial_{e_{l}}\left(s_{l}\right)_{\mid \tau_{l}}\left(\tau_{l}, e_{l}\right)=\partial_{e_{g}}\left(s_{g}\right)_{\mid \tau_{g}}\left(\tau_{g}, e_{g}\right) \\
s_{l}\left(\tau_{l}, e_{l}\right)-\tau \partial_{e_{l}}\left(s_{l}\right)_{\mid \tau_{l}}\left(\tau_{l}, e_{l}\right)-e_{l} \partial_{\tau_{l}}\left(s_{l}\right)_{\mid e_{l}}\left(\tau_{l}, e_{l}\right)=s_{g}\left(\tau_{g}, e_{g}\right)-\tau \partial_{e_{g}}\left(s_{g}\right)_{\mid \tau_{g}}\left(\tau_{g}, e_{g}\right)-e_{g} \partial_{\tau_{g}}\left(s_{g}\right)_{\mid e_{g}}\left(\tau_{g}, e_{g}\right) .
\end{array}\right.
$$

Moreover, if the maximizer $Z_{g l}$ belongs to the degeneracy manifold of $\widetilde{\eta}$ condition (3.24) holds. When $\alpha_{k} \neq 0$ and $m_{k} \neq 0$, this condition can be expressed in terms of the densities $\rho_{k}=m_{k} / \alpha_{k}$ and the internal energies $e_{k}=\varepsilon_{l} / m_{l}$ :

$$
\left\{\begin{array} { l } 
{ \kappa \alpha _ { g } = \alpha _ { l } } \\
{ \kappa \alpha _ { g } \rho _ { g } = \alpha _ { l } \rho _ { l } } \\
{ \kappa \alpha _ { g } \rho _ { g } e _ { g } = \alpha _ { l } \rho _ { l } e _ { l } }
\end{array} \Longleftrightarrow \left\{\begin{array}{l}
\kappa \alpha_{g}=\alpha_{l} \\
\rho_{g}=\rho_{l} \\
e_{g}=e_{l}
\end{array} .\right.\right.
$$

Then, if we introduce in relations (3.29) the equalities $\tau=\tau_{l}=\tau_{g}$ and $e=e_{l}=e_{g}$ arising from (3.30), we get the relations:

$$
\left\{\begin{array}{l}
\partial_{\tau_{l}}\left(s_{l}\right)_{\mid e_{l}}(\tau, e)=\partial_{\tau_{g}}\left(s_{g}\right)_{\mid e_{g}}(\tau, e), \\
\partial_{e_{l}}\left(s_{l}\right)_{\mid \tau_{l}}(\tau, e)=\partial_{e_{g}}\left(s_{g}\right)_{\mid \tau_{g}}(\tau, e), \\
s_{l}(\tau, e)=s_{g}(\tau, e) .
\end{array}\right.
$$

Hence, if the phasic entropies are chosen so that condition (3.25) holds, relations (3.31) cannot be fulfilled. In other words, when the first derivatives of $\widetilde{\eta}$ vanish at a given point, the latter does not belong to the degeneracy manifold. This means that even if the entropy $\widetilde{\eta}$ is not strictly concave, it possesses a unique maximum on $\widetilde{H}_{\eta, 0}$.

In order to obtain this result, we have assumed that $\alpha_{k} \neq 0$ and $m_{k} \neq 0$. This is the case thanks to the definition of $H_{\eta}$ for which single phase situations have been excluded, see also the remark at the end of Section 2. Finally, if single-phase flows situations do not occur, the entropy $\widetilde{\eta}$ has a unique maximum on $\widetilde{H}_{\eta, 0}$. This ends the proof of Corollary 3.4.

Remark 3.5. It should be noticed that condition (3.25) on the phasic entropies is not too restrictive. In practice, in order to enforce the system to avoid single-phase flow situations, the entropies could be chosen so that:

$$
\begin{aligned}
& \forall m_{k}>0, \forall \varepsilon_{k}>0, m_{k} s_{k}\left(\frac{\alpha_{k}}{m_{k}}, \frac{\varepsilon_{k}}{m_{k}}\right) \underset{\alpha_{k} \rightarrow 0^{+}}{\longrightarrow}-\infty, \\
& \forall \alpha_{k}>0, \forall \varepsilon_{k}>0, m_{k} s_{k}\left(\frac{\alpha_{k}}{m_{k}}, \frac{\varepsilon_{k}}{m_{k}}\right) \underset{m_{k} \rightarrow 0^{+}}{\longrightarrow}-\infty, \\
& \forall \alpha_{k}>0, \forall m_{k}>0, m_{k} s_{k}\left(\frac{\alpha_{k}}{m_{k}}, \frac{\varepsilon_{k}}{m_{k}}\right) \underset{\varepsilon_{k} \rightarrow 0^{+}}{\longrightarrow}-\infty .
\end{aligned}
$$


The first and third conditions of (3.32) are classical, but in general the second one is not fulfilled (for instance for perfect gas EOS). There is indeed no physical reason to prevent a phase to vanish when mass transfer is accounted for. Vanishing phases treatment is a tricky problem for two-fluid models.

\subsection{Definition of entropies for the complete model}

Let us introduce the vectors of conservative variables $W_{k}=\left(\alpha_{k}, m_{k}, \mathcal{E}_{k}, Q_{k}\right) \in H_{s, k}$ with the domain $H_{s, k}=$ $\left(H_{\eta, k} \times \mathbb{R}\right)$ and $W_{g l}=\left(\alpha_{g}, m_{g}, \mathcal{E}_{g}, Q_{g}, \alpha_{l}, m_{l}, \mathcal{E}_{l}, Q_{l}\right) \in H_{s}$ with the domain $H_{s}=\left(H_{\eta, g} \times \mathbb{R} \times H_{\eta, l} \times \mathbb{R}\right)$. We define the following entropies for the variables $W_{k}$ and $W_{g l}$ :

$$
\mathcal{S}_{k}:\left(\begin{array}{l}
H_{s, k} \rightarrow \mathbb{R} \\
W_{k} \mapsto \eta_{k}\left(\alpha_{k}, m_{k}, \mathcal{E}_{k}-\frac{Q_{k}^{2}}{2 m_{k}}\right)
\end{array}\right),
$$

and

$$
\mathcal{S}_{g l}:\left(\begin{array}{l}
H_{s} \rightarrow \mathbb{R} \\
W_{g l} \mapsto \mathcal{S}_{g}\left(\alpha_{g}, m_{g}, \mathcal{E}_{g}, Q_{g}\right)+\mathcal{S}_{l}\left(\alpha_{l}, m_{l}, \mathcal{E}_{l}, Q_{l}\right)
\end{array}\right) .
$$

The entropy $\mathcal{S}_{g l}$ is thus the sum of the phasic entropies $\mathcal{S}_{k}$ and it accounts for the whole set of the conservative variables $W_{g l}$ of system $(2.1)$.

Proposition 3.6. The mixture entropy $W_{g l} \mapsto \mathcal{S}\left(W_{g l}\right)$ has the following properties.

- $\forall a \in \mathbb{R}_{*}^{+}, \forall W \in H_{s}, \mathcal{S}(a W)=a \mathcal{S}(W) ;$

- $\mathcal{S}$ belongs to $C^{2}\left(H_{s}, \mathbb{R}\right)$;

- $W_{g l} \mapsto \mathcal{S}\left(W_{g l}\right)$ is concave on $H_{s}$, its degeneracy manifold is:

$$
\mathcal{M}_{S}\left(W_{g l}\right)=\left\{a\left(\alpha_{g}, m_{g}, \mathcal{E}_{g}, Q_{g}, 0,0,0,0\right)+b\left(0,0,0,0, \alpha_{l}, m_{l}, \mathcal{E}_{l}, Q_{l}\right),(a, b) \in \mathbb{R}^{2}\right\} .
$$

Proof. We proceed here using a separation of variables as in the proof of Proposition 3.1. The first and second properties are directly inherited from the properties of the phasic entropies $\eta_{k}$. Let us focus on the third property. For that purpose, we remark that for all $\left(w_{k}, x_{k}, y_{k}, z_{k}\right) \in \mathbb{R}^{4}$ we can obtain for $\mathcal{S}_{k}^{\prime \prime}$, the Hessian matrix of $W_{k} \mapsto \mathcal{S}_{k}\left(W_{k}\right)$, the relation:

$$
\begin{aligned}
\left(w_{k}, x_{k}, y_{k}, z_{k}\right) \cdot \mathcal{S}_{k}^{\prime \prime} \cdot\left(\begin{array}{l}
w_{k} \\
x_{k} \\
y_{k} \\
z_{k}
\end{array}\right)= & \left(w_{k}, x_{k}, x_{k} \frac{Q_{k}^{2}}{2 m_{k}^{2}}+y_{k}-z_{k} \frac{Q_{k}}{m_{k}}\right) \cdot \eta_{k}^{\prime \prime} \cdot\left(\begin{array}{c}
w_{k} \\
x_{k} \\
x_{k} \frac{Q_{k}^{2}}{2 m_{k}^{2}}+y_{k}-z_{k} \frac{Q_{k}}{m_{k}}
\end{array}\right) \\
& -\frac{\left(x_{k} \frac{Q_{k}}{m_{k}}-z_{k}\right)^{2}}{m_{k}} \partial_{\varepsilon_{k}}\left(\eta_{k}\right)_{\mid \alpha_{k}, m_{k}} .
\end{aligned}
$$

For the sake of readability, the details of the derivatives are reported in Appendix A. Thanks to the third item of Definition 2.1 and to relation (3.7), the second term on the right hand side of relation (3.36) is negative. Moreover, the property of concavity of $\eta_{k}$ reported in Proposition 3.1 ensures that the first term on the right hand side of relation (3.36) is also negative. Hence,

$$
\left(w_{k}, x_{k}, y_{k}, z_{k}\right) \in \mathbb{R}^{4},\left(w_{k}, x_{k}, y_{k}, z_{k}\right) \cdot \mathcal{S}_{k}^{\prime \prime} \cdot\left(\begin{array}{c}
w_{k} \\
x_{k} \\
y_{k} \\
z_{k}
\end{array}\right) \leq 0
$$

which means that the phasic entropy $W_{k} \mapsto \mathcal{S}_{k}\left(W_{k}\right)$ is concave on $H_{s, k}$. Thanks to the separation of variables in the definition of $\mathcal{S}$, it is easily obtained that for $\mathcal{Y}=\left(w_{g}, x_{g}, y_{g}, z_{g}, w_{l}, x_{l}, y_{l}, z_{l}\right) \in \mathbb{R}^{8}$ the Hessian matrix $\mathcal{S}^{\prime \prime}$ 
of the entropy $W_{g l} \mapsto \mathcal{S}\left(W_{g l}\right)$ fulfills the relation:

$$
\begin{aligned}
\mathcal{Y} \cdot \mathcal{S}^{\prime \prime} \cdot \mathcal{Y}^{\top}= & \sum_{k}\left(\left(w_{k}, x_{k}, x_{k} \frac{Q_{k}^{2}}{2 m_{k}^{2}}+y_{k}-z_{k} \frac{Q_{k}}{m_{k}}\right) \cdot \eta_{k}^{\prime \prime} \cdot\left(\begin{array}{c}
w_{k} \\
x_{k} \\
x_{k} \frac{Q_{k}^{2}}{2 m_{k}^{2}}+y_{k}-z_{k} \frac{Q_{k}}{m_{k}}
\end{array}\right)\right) \\
& -\sum_{k}\left(\frac{\left(x_{k} \frac{Q_{k}}{m_{k}}-z_{k}\right)^{2}}{m_{k}} \partial_{\varepsilon_{k}}\left(\eta_{k}\right)_{\mid \alpha_{k}, m_{k}}\right) .
\end{aligned}
$$

All the terms on the right hand side of relation (3.37) are negative, we can thus conclude that $W_{g l} \mapsto \mathcal{S}\left(W_{g l}\right)$ is concave on $H_{s}$. Moreover, the degeneracy manifold $\mathcal{M}_{s}\left(W_{g l}\right)$ of $\mathcal{S}$ at a point $W_{g l} \in H_{s}$ can be obtained as the set of vectors $\mathcal{Y}$ such that: $\mathcal{Y} \cdot \mathcal{S}^{\prime \prime} \cdot \mathcal{Y}^{\top}=0$. Since all the terms on the right hand side of relation (3.37) are negative, this is equivalent to:

$$
\left\{\begin{array}{c}
\left(w_{g}, x_{g}, x_{g} \frac{Q_{g}^{2}}{2 m_{g}^{2}}+y_{g}-z_{g} \frac{Q_{g}}{m_{g}}, w_{l}, x_{l}, x_{l} \frac{Q_{l}^{2}}{2 m_{l}^{2}}+y_{l}-z_{l} \frac{Q_{l}}{m_{l}}\right) \in \mathcal{M}_{\eta}\left(\left(\alpha_{g}, m_{g}, \mathcal{E}_{g}-\frac{Q_{g}^{2}}{2 m_{g}}, \alpha_{l}, m_{l}, \mathcal{E}_{l}-\frac{Q_{l}^{2}}{2 m_{l}}\right)\right) \\
\text { and }\left\{\begin{array}{c}
x_{g} \frac{Q_{g}}{m_{g}}-z_{g}=0 \\
x_{l} \frac{Q_{l}}{m_{l}}-z_{l}=0
\end{array}\right.
\end{array}\right.
$$

By using substitutions, this set of eight linear equations leads to:

$$
\mathcal{M}_{S}\left(W_{g l}\right)=\left\{a\left(\alpha_{g}, m_{g}, \mathcal{E}_{g}, Q_{g}, 0,0,0,0\right)+b\left(0,0,0,0, \alpha_{l}, m_{l}, \mathcal{E}_{l}, Q_{l}\right),(a, b) \in \mathbb{R}^{2}\right\} .
$$

The degeneracy manifold $\mathcal{M}_{S}\left(W_{g l}\right)$ is thus a subspace of dimension 2 of $\mathbb{R}^{8}$. This ends the proof of Proposition 3.6.

All the first and second derivatives of the entropy $\mathcal{S}_{k}$ are reported in Appendix A. The second derivative of $\mathcal{S}_{k}$ with respect to the mass $m_{k}$ is given by equation (A.14). Thanks to the properties of the entropies $\eta_{k}$ the following proposition can be stated.

Proposition 3.7. The function $m_{k} \mapsto \mathcal{S}_{m, k}\left(m_{k}\right)$ defined for given $\alpha_{k} \in \mathbb{R}_{*}^{+}, \mathcal{E}_{k} \in \mathbb{R}_{*}^{+}$and $Q_{k} \in \mathbb{R}_{*}^{+}$by:

$$
\mathcal{S}_{m, k}:\left(\begin{array}{l}
\mathbb{R}_{*}^{+} \rightarrow \mathbb{R} \\
m_{k} \mapsto \mathcal{S}_{k}\left(\alpha_{k}, m_{k}, \mathcal{E}_{k}, Q_{k}\right)
\end{array}\right),
$$

belongs to $C^{2}\left(\mathbb{R}_{*}^{+}, \mathbb{R}\right)$ and it is strictly concave on $\mathbb{R}_{*}^{+}$.

Proof. Since the entropy $\eta_{k}$ belongs to $C^{2}\left(H_{\eta, k}, \mathbb{R}\right), m_{k} \mapsto \mathcal{S}_{m, k}\left(m_{k}\right)$ obviously belongs to $C^{2}\left(\mathbb{R}_{*}^{+}, \mathbb{R}\right)$. From the Appendix A, the second derivative of $m_{k} \mapsto \mathcal{S}_{m, k}\left(m_{k}\right)$ is given by equation (A.14). We then have:

$$
\mathcal{S}_{m, k}^{\prime \prime}\left(m_{k}\right)=-\frac{Q_{k}^{2}}{m_{k}^{3}} \partial_{\varepsilon_{k}}\left(\eta_{k}\right)_{\mid \alpha_{k}, m_{k}}+\left(1, \frac{Q_{k}^{2}}{2 m_{k}}\right) \cdot\left(\begin{array}{cc}
\partial_{m_{k}, m_{k}}^{2}\left(\eta_{k}\right) & \partial_{m_{k}, \varepsilon_{k}}^{2}\left(\eta_{k}\right) \\
\partial_{m_{k}, \varepsilon_{k}}^{2}\left(\eta_{k}\right) & \partial_{\varepsilon_{k}, \varepsilon_{k}}^{2}\left(\eta_{k}\right)
\end{array}\right) \cdot\left(\begin{array}{c}
1 \\
\frac{Q_{k}^{2}}{2 m_{k}}
\end{array}\right) .
$$

Thanks to equation (3.7) and to the property of $s_{k}$ (see Def. 2.1), we have $\partial_{\varepsilon_{k}}\left(\eta_{k}\right)_{\mid \alpha_{k}, m_{k}}=1 / T_{k}>0$, and therefore the first term on the right hand side of relation (3.39) is non-positive. In the other hand, $Z_{k} \mapsto \eta_{k}\left(Z_{k}\right)$ is concave on $H_{\eta, k}$, hence the function which for a given $\alpha_{k} \in \mathbb{R}_{*}^{+}$is defined by $\left(m_{k}, \varepsilon\right) \mapsto \eta_{k}\left(\alpha_{k}, m_{k}, \varepsilon\right)$ is also concave. As a consequence, the matrix in the second term on the right hand side of equation (3.39) is symmetric definite negative. This allows to conclude that:

$$
\forall m_{k} \in \mathbb{R}_{*}^{+}, \mathcal{S}_{m, k}^{\prime \prime}\left(m_{k}\right)<0 .
$$

This ends the proof of Proposition 3.7. 
The entropy $W_{g l} \mapsto \mathcal{S}\left(W_{g l}\right)$ is concave on $H_{s}$ but it is not strictly concave since its degeneracy manifold $\mathcal{M}_{S}\left(W_{g l}\right)$ is a vector subspace of dimension 2 of $\mathbb{R}^{8}$. We define now the entropy $\widetilde{\mathcal{S}}$ that is the restriction of $\mathcal{S}$ on $\widetilde{H}_{s, 0}$, a subset of $H_{s}$ :

$$
\widetilde{\mathcal{S}}:\left(\begin{array}{l}
\widetilde{H}_{s, 0} \rightarrow \mathbb{R} \\
W_{g l} \mapsto \mathcal{S}\left(W_{g l}\right)
\end{array}\right)
$$

The domain $\widetilde{H}_{s, 0}$ is the subset of $H_{s}$ that corresponds to a given sum for the fractions $\alpha_{g}+\alpha_{l}=\alpha_{0}$, for the partial masses $m_{g}+m_{l}=m_{0}$, for the total energies $\mathcal{E}_{g}+\mathcal{E}_{l}=\mathcal{E}_{0}$ and for the momentum $Q_{g}+Q_{l}=Q_{0}$ :

$$
\widetilde{H}_{s, 0}=\left\{W_{g l} \in H_{s} ; \alpha_{g}+\alpha_{l}=\alpha_{0}, m_{g}+m_{l}=m_{0}, \mathcal{E}_{g}+\mathcal{E}_{l}=\mathcal{E}_{0}, Q_{g}+Q_{l}=Q_{0}\right\} .
$$

It can easily be shown that this domain $\widetilde{H}_{s, 0}$ is a convex subset of $\left(\left(\mathbb{R}^{+}\right)^{3} \times \mathbb{R}\right)^{2}$. With this restriction of the domain of definition, the entropy $\widetilde{\mathcal{S}}$ has the following property.

Theorem 3.8. The entropy $W_{g l} \mapsto \widetilde{\mathcal{S}}\left(W_{g l}\right)$ defined by (3.40) and (3.41) is strictly concave on $\widetilde{H}_{s, 0}$, except at the points $W_{\text {gl }}$ for which there exists $\kappa \in \mathbb{R}_{*}^{+}$such that:

$$
\left\{\begin{array}{l}
\kappa \alpha_{g}=\alpha_{l} \\
\kappa m_{g}=m_{l} \\
\kappa \mathcal{E}_{g}=\mathcal{E}_{l} \\
\kappa Q_{g}=Q_{l}
\end{array}\right.
$$

In such situations, the degeneracy manifold of $W_{g l} \mapsto \widetilde{\mathcal{S}}\left(W_{\text {gl }}\right)$ is the sub-space of $\mathbb{R}^{8}$ :

$$
\mathcal{M}_{S}\left(W_{g l}\right) \cap \widetilde{H}_{s, 0}=\left\{\left(\alpha_{0}, m_{0}, \mathcal{E}_{0}, Q_{0}, 0,0,0,0\right)+b\left(-\alpha_{l},-m_{l},-\mathcal{E}_{l},-Q_{l}, \alpha_{l}, m_{l}, \mathcal{E}_{l}, Q_{l}\right), \quad b \in \mathbb{R}\right\} .
$$

Proof. From Proposition 3.6, we can deduce that $W_{g l} \mapsto \widetilde{\mathcal{S}}\left(W_{g l}\right)$ is concave on $\widetilde{H}_{s, 0} \subset H_{s}$. In order to exhibit the form of the degeneracy manifold (3.42), let us choose a point which belongs to the degeneracy maniflod of $\widetilde{\mathcal{S}}$ and to $\widetilde{H}_{s, 0}$ :

$$
W_{g l}=\left(\alpha_{g}, m_{g}, \mathcal{E}_{g}, Q_{g}, \alpha_{l}, m_{l}, \mathcal{E}_{l}, Q_{l}\right) \in \widetilde{H}_{s, 0}
$$

By definition of $\mathcal{M}_{S}\left(W_{g l}\right)$ and $\widetilde{H}_{s, 0}$, there exist $(a, b) \in \mathbb{R}^{2}$ such that:

$$
\left\{\begin{array}{l}
\alpha_{g}+\alpha_{l}=a \alpha_{g}+b \alpha_{l}=\alpha_{0} \\
m_{g}+m_{l}=a m_{g}+b m_{l}=m_{0} \\
\mathcal{E}_{g}+\mathcal{E}_{l}=a \mathcal{E}_{g}+b \mathcal{E}_{l}=\mathcal{E}_{0} \\
Q_{g}+Q_{l}=a Q_{g}+b Q_{l}=m_{0}
\end{array}\right.
$$

This system of equations leads to the system:

$$
\left\{\begin{array}{l}
(a-1) \alpha_{g}+(b-1) \alpha_{l}=0 \\
(a-1) m_{g}+(b-1) m_{l}=0 \\
(a-1) \mathcal{E}_{g}+(b-1) \mathcal{E}_{l}=0 \\
(a-1) Q_{g}+(b-1) Q_{l}=0
\end{array}\right.
$$

If at least two among the four equations of system (3.43) are linearly independent, there exists a unique solution $(a, b)$ to system (3.43): $a=b=1$. Hence, in such a situation, the intersection of the degeneracy manifold $\mathcal{M}_{S}\left(W_{g l}\right)$ with the domain $\widetilde{H}_{s, 0}$ is restricted to a single point:

$$
\mathcal{M}_{S}\left(W_{g l}\right) \cap \widetilde{H}_{s, 0}=\left\{W_{g l}\right\}
$$


The entropy $W_{g l} \mapsto \widetilde{\mathcal{S}}\left(W_{g l}\right)$ is then strictly concave. On the contrary, when the four equations of (3.43) are equivalent, the solution $(a, b)$ is not unique. This situation occurs when there exists $\kappa \in \mathbb{R}_{*}^{+}$such that:

$$
\left\{\begin{array}{l}
\kappa \alpha_{g}=\alpha_{l} \\
\kappa m_{g}=m_{l} \\
\kappa \mathcal{E}_{g}=\mathcal{E}_{l} \\
\kappa Q_{g}=Q_{l} .
\end{array}\right.
$$

and system (3.43) leads to the relation:

$$
a=1+(1-b) \kappa .
$$

In such a situation, the entropy $W_{g l} \mapsto \widetilde{\mathcal{S}}\left(W_{g l}\right)$ is not strictly concave and its degeneracy manifold is the sub-space of $\mathbb{R}^{8}$ :

$$
\mathcal{M}_{S}\left(W_{g l}\right) \cap \widetilde{H}_{s, 0}=\left\{\left(\alpha_{0}, m_{0}, \mathcal{E}_{0}, Q_{0}, 0,0,0,0\right)+b\left(-\alpha_{l},-m_{l},-\mathcal{E}_{l},-Q_{l}, \alpha_{l}, m_{l}, \mathcal{E}_{l}, Q_{l}\right), b \in \mathbb{R}_{*}^{+}\right\} .
$$

This ends the proof of Theorem 3.8.

\section{Classical Closure laws for the source terms}

In this section, we recall the classical approach used to build some admissible closure laws for the source terms. The latter is widely used and several references deal with such source terms, see $[1,5,6,12]$ among many others. The source terms $S_{i, k}, i=\{1,2,3,4\}, k=\{l, g\}$, are first decomposed into elementary effects. The momentum exchange source term $S_{3, k}$ is split into a contribution due to the drag force $D_{u, k}$ and a contribution due to the mass exchange $S_{2, k}$ :

$$
S_{3, k}=D_{u, k}+\tilde{V}\left(W_{g l}\right) S_{2, k}
$$

with $D_{u, g}=-D_{u, l}$. In a similar way, its is assumed that the energy exchange source term $S_{4, k}$ gathers a "pure" heat exchange term $\Psi_{k}$, a contribution due the exchange of energy associated with the drag force and the heat exchange due to the mass transfer:

$$
S_{4, k}=\Psi_{k}+\bar{V}\left(W_{g l}\right) D_{u, k}+\bar{H}\left(W_{g l}\right) S_{2, k}
$$

with $\Psi_{g}=-\Psi_{l}$. The velocity terms $\left(W_{g l}\right) \mapsto \widetilde{V}\left(W_{g l}\right)$ and $\left(W_{g l}\right) \mapsto \bar{V}\left(W_{g l}\right)$, and the enthalpy term $\left(W_{g l}\right) \mapsto$ $\bar{H}\left(W_{g l}\right)$ have to be closed. These choices are in agreement with conservation constraint (2.2). Equation (2.11) for the specific entropy can then be written:

$$
\begin{aligned}
\partial_{t}\left(m_{k} s_{k}\right)+\partial_{x}\left(m_{k} U_{k} s_{k}\right)= & \frac{\left(U_{k}-V_{I}\right)\left(P_{k}-P_{I}\right)}{T_{k}} \partial_{x}\left(\alpha_{k}\right) \\
& +\frac{\left(P_{k}-P_{I}\right)}{T_{k}} S_{1, k}+\frac{-\mu_{k}+\bar{H}-U_{k} \widetilde{V}-U_{k}^{2} / 2}{T_{k}} S_{2, k}+\frac{\bar{V}-U_{k}}{T_{k}} D_{u, k}+\frac{1}{T_{k}} \Psi_{k} .
\end{aligned}
$$

By summing the two equations (4.1), the terms of equation (4.1) that contain the derivative with respect to the fraction $\alpha_{k}$ vanish and we obtain:

$$
\begin{aligned}
\partial_{t}\left(m_{g} s_{g}+m_{l} s_{l}\right)+\partial_{x}\left(m_{g} U_{g} s_{g}+m_{l} U_{l} s_{l}\right)= & \frac{\left(P_{g}-P_{l}\right)}{(1-\beta) T_{g}+\beta T_{l}} S_{1, g} \\
& +\left(\frac{\mu_{l}}{T_{l}}-\frac{\mu_{g}}{T_{g}}+\frac{\bar{H}-U_{g} \widetilde{V}-U_{g}^{2} / 2}{T_{g}}-\frac{\bar{H}-U_{l} \widetilde{V}-U_{l}^{2} / 2}{T_{l}}\right) S_{2, g} \\
& +\left(\frac{\bar{V}-U_{g}}{T_{g}}-\frac{\bar{V}-U_{l}}{T_{l}}\right) D_{u, g}+\left(\frac{1}{T_{g}}-\frac{1}{T_{l}}\right) \Psi_{g} .
\end{aligned}
$$


The classical approach then considers the thermodynamical entropy $\eta=\left(m_{g} s_{g}+m_{l} s_{l}\right)$ and the associated entropy-flux $F_{\eta}=\left(\eta_{g} U_{g}+\eta_{l} U_{l}\right)$ (see definitions (3.1) and (3.3)). Since the entropy $\eta$ is concave (see Sect. 3), and in order to agree with the second law of thermodynamics, the admissible closure laws for the source terms $S_{1, g}, S_{2, g}, D_{u, g}$ and $\Psi_{g}$ must fulfill the entropy inequality:

$$
\partial_{t}(\eta)+\partial_{x}\left(F_{\eta}\right) \geq 0
$$

The source terms $S_{1, g}, S_{2, g}, D_{u, g}$ and $\Psi_{g}$ are thus not defined in a unique manner, but a classical choice is to choose closures that ensure the positivity of each terms in the right hand side of (4.2):

$$
\begin{aligned}
\left(\frac{\mu_{l}}{T_{l}}-\frac{\mu_{g}}{T_{g}}+\frac{\bar{H}-U_{g} \widetilde{V}-U_{g}^{2} / 2}{T_{g}}-\frac{\left.\bar{H}-U_{l}-P_{l}\right)}{T_{l}-U_{l}^{2} / 2}\right) S_{1, g} & \geq 0 \\
T_{l} & \geq 0 \\
\left(\frac{\bar{V}-U_{g}}{T_{g}}-\frac{\bar{V}-U_{l}}{T_{l}}\right) D_{u, g} & \geq 0 \\
\left(\frac{1}{T_{g}}-\frac{1}{T_{l}}\right) \Psi_{g} & \geq 0 .
\end{aligned}
$$

A simple manner is then to write the following closure laws:

$$
\left\{\begin{aligned}
S_{1, g} & =K_{1}\left(W_{g l}\right)\left(P_{g}-P_{l}\right) \\
S_{2, g} & =K_{2}\left(W_{g l}\right)\left(\frac{\mu_{l}}{T_{l}}-\frac{\mu_{g}}{T_{g}}+\frac{\bar{H}-U_{g} \tilde{V}-U_{g}^{2} / 2}{T_{g}}-\frac{\bar{H}-U_{l} \tilde{V}-U_{l}^{2} / 2}{T_{l}}\right) \\
D_{u, g} & =K_{3}\left(W_{g l}\right)\left(\frac{\bar{V}-U_{g}}{T_{g}}-\frac{\bar{V}-U_{l}}{T_{l}}\right) \\
\Psi_{g} & =K_{4}\left(W_{g l}\right)\left(\frac{1}{T_{g}}-\frac{1}{T_{l}}\right)
\end{aligned}\right.
$$

where the functions $W_{g l} \mapsto K_{i}\left(W_{g l}\right), i=\{1,2,3,4\}$, can depend on the variable $W_{g l}$ but they have to be positive:

$$
\forall i=\{1,2,3,4\}, \forall W_{g l}, K_{i}\left(W_{g l}\right) \geq 0 .
$$

It should be noted that no constraint arises on the terms $\widetilde{V}, \bar{V}$ and $\bar{H}$. Nevertheless, the closure $\bar{V}=\left(U_{g}+U_{l}\right) / 2$ allows to retrieve a classical admissible form for the drag force based on the relative velocity:

$$
D_{u, g}=K_{3}\left(W_{g l}\right)\left(\frac{1}{2 T_{g}}+\frac{1}{2 T_{l}}\right)\left(U_{l}-U_{g}\right)=K_{3, u}\left(W_{g l}\right)\left(U_{l}-U_{g}\right) .
$$

It can also be noted that with the choice $\widetilde{V}=\bar{V}=\left(U_{g}+U_{l}\right) / 2$ and $\bar{H}=U_{g} U_{l} / 2$, the mass transfer term $S_{2, g}$ can be simplified in:

$$
S_{2, g}=K_{2}\left(W_{g l}\right)\left(\frac{\mu_{l}}{T_{l}}-\frac{\mu_{g}}{T_{g}}\right) .
$$

Thus the mass transfer does not depend on the velocities of the phases, and is only related to the difference between the chemical potentials $\mu_{k} / T_{k}$. Obviously, other modeling choices can be considered.

\section{Non-Classical Closure laWs FOR THE SOURCE TERMS}

The set of closure laws proposed in this section are based on the approach mainly used in the modeling of multiphase flows using single-velocity models, as in $[2,10,19,20]$ for instance. In Section 3 , the different entropies have been studied regardless of the set of partial derivative equations (2.1) that defines the paths followed by the different variables. In order to reintroduce this information, let us first write system (2.1) in slightly different but 
equivalent form. The time derivative of the fraction $\alpha_{k}$ in the energy equation is replaced by a space derivative by using first equation of (2.1). This leads to the system of equations:

$$
\left\{\begin{array}{l}
\partial_{t}\left(\alpha_{g}\right)+V_{I} \partial_{x}\left(\alpha_{g}\right)=S_{1, g}^{\prime} \\
\partial_{t}\left(\alpha_{g} \rho_{g}\right)+\partial_{x}\left(\alpha_{g} \rho_{g} U_{g}\right)=S_{2, g}^{\prime} \\
\partial_{t}\left(\alpha_{g} \rho_{g} U_{g}\right)+\partial_{x}\left(\alpha_{g} \rho_{g} U_{g}^{2}+\alpha_{g} P_{g}\right)-P_{I} \partial_{x}\left(\alpha_{g}\right)=S_{3, g}^{\prime} \\
\partial_{t}\left(\alpha_{g} \rho_{g} E_{g}\right)+\partial_{x}\left(\alpha_{g} U_{g}\left(\rho_{g} E_{g}+P_{g}\right)\right)-P_{I} V_{I} \partial_{x}\left(\alpha_{g}\right)=S_{4, g}^{\prime} \\
\partial_{t}\left(\alpha_{l} \rho_{l}\right)+\partial_{x}\left(\alpha_{l} \rho_{l} U_{l}\right)=S_{2, l} \\
\partial_{t}\left(\alpha_{l} \rho_{l} U_{l}\right)+\partial_{x}\left(\alpha_{l} \rho_{l} U_{l}^{2}+\alpha_{l} P_{l}\right)-P_{I} \partial_{x}\left(\alpha_{l}\right)=S_{3, l}^{\prime} \\
\partial_{t}\left(\alpha_{l} \rho_{l} E_{l}\right)+\partial_{x}\left(\alpha_{l} U_{l}\left(\rho_{l} E_{l}+P_{l}\right)\right)-P_{I} V_{I} \partial_{x}\left(\alpha_{l}\right)=S_{4, l}^{\prime} .
\end{array}\right.
$$

The source terms are for $k=\{l, g\}: S_{1, k}^{\prime}=S_{1, k}, S_{2, k}^{\prime}=S_{2, k}, S_{3, k}^{\prime}=S_{3, k}$, but with $S_{4, k}^{\prime}=S_{4, k}-P_{I} S_{1, k}$. We then also get the property conservation for an isolated system:

$$
\forall i=\{1,2,3,4\}, S_{i, l}^{\prime}+S_{i, g}^{\prime}=0
$$

All the properties that have been recalled in Section 2 for system (2.1) hold for system (5.1). In fact, system (5.1) is just a more convenient form of the two-fluid model when considering the source terms of the present section. Equation (2.11) on the phasic entropy $s_{k}$ becomes for system (5.1):

$$
\begin{aligned}
\partial_{t}\left(m_{k} s_{k}\right)+\partial_{x}\left(m_{k} U_{k} s_{k}\right)= & \frac{\left(U_{k}-V_{I}\right)\left(P_{k}-P_{I}\right)}{T_{k}} \partial_{x}\left(\alpha_{k}\right) \\
& +\frac{P_{k}}{T_{k}} S_{1, k}^{\prime}+\frac{-\mu_{k}-U_{k}^{2} / 2}{T_{k}} S_{2, k}^{\prime}-\frac{U_{k}}{T_{k}} S_{3, k}^{\prime}+\frac{1}{T_{k}} S_{4, k}^{\prime} .
\end{aligned}
$$

The equation for the mixture entropy $\widetilde{\mathcal{S}}$ is then obtained by summing equations (5.3) and by applying the constraints $\alpha_{l}+\alpha_{g}=1$ and $S_{i, l}^{\prime}+S_{i, g}^{\prime}=0$, which are associated with the domain $\widetilde{H}_{s, 0}$. If the closures for $V_{I}$ (2.15) and $P_{I}(2.16)$ are retained, we get:

$$
\begin{aligned}
\partial_{t}(\widetilde{\mathcal{S}})+\partial_{x}\left(\frac{\mathcal{Q}_{l}}{m_{l}} \mathcal{S}_{l}+\frac{\mathcal{Q}_{g}}{m_{g}} \mathcal{S}_{g}\right)= & \left(\frac{P_{g}}{T_{g}}-\frac{P_{l}}{T_{l}}\right) S_{1, g}^{\prime}+\left(\frac{-\mu_{g}-U_{g}^{2} / 2}{T_{g}}-\frac{-\mu_{l}-U_{l}^{2} / 2}{T_{l}}\right) S_{2, g}^{\prime} \\
& +\left(\frac{U_{l}}{T_{l}}-\frac{U_{g}}{T_{g}}\right) S_{3, g}^{\prime}+\left(\frac{1}{T_{g}}-\frac{1}{T_{l}}\right) S_{4, g}^{\prime} .
\end{aligned}
$$

Let us now express the derivative of $W_{g l} \mapsto \widetilde{\mathcal{S}}\left(W_{g l}\right)$ with respect to $\alpha_{g}$ on $\widetilde{H}_{s, 0}$, where the fraction $\alpha_{0}$ in definition (3.41) is equal to 1 :

$$
\begin{aligned}
\left.\partial_{\alpha_{g}}(\widetilde{\mathcal{S}})\right|_{\alpha_{l}+\alpha_{g}=1, m_{k}, \mathcal{Q}_{k}, \mathcal{E}_{k}} & =\left.\partial_{\alpha_{g}}\left(\mathcal{S}_{g}\right)\right|_{m_{g}, \mathcal{Q}_{g}, \mathcal{E}_{g}}+\left.\partial_{\alpha_{g}}\left(\mathcal{S}_{l}\right)\right|_{\alpha_{l}+\alpha_{g}=1, m_{l}, \mathcal{Q}_{l}, \mathcal{E}_{l}} \\
& =\left.\partial_{\alpha_{g}}\left(\mathcal{S}_{g}\right)\right|_{m_{g}, \mathcal{Q}_{g}, \mathcal{E}_{g}}+\left.\left.\partial_{\alpha_{g}}\left(\alpha_{l}\right)\right|_{\alpha_{l}+\alpha_{g}=1} \partial_{\alpha_{l}}\left(\mathcal{S}_{l}\right)\right|_{m_{l}, \mathcal{Q}_{l}, \mathcal{E}_{l}} \\
& =\left.\partial_{\alpha_{g}}\left(\mathcal{S}_{g}\right)\right|_{m_{g}, \mathcal{Q}_{g}, \mathcal{E}_{g}}-\left.\partial_{\alpha_{l}}\left(\mathcal{S}_{l}\right)\right|_{m_{l}, \mathcal{Q}_{l}, \mathcal{E}_{l}} \\
& =\frac{P_{g}}{T_{g}}-\frac{P_{l}}{T_{l}}
\end{aligned}
$$


The last formula of the set of relations above is obtained thanks to relation (A.5) of appendix A. In a similar way, one can easily show that:

$$
\begin{aligned}
\left.\partial_{m_{g}}(\widetilde{\mathcal{S}})\right|_{m_{l}+m_{g}=m_{0}, \alpha_{k}, \mathcal{Q}_{k}, \mathcal{E}_{k}} & =\frac{-\mu_{g}-U_{g}^{2} / 2}{T_{g}}-\frac{-\mu_{l}-U_{l}^{2} / 2}{T_{l}} \\
\left.\partial_{\mathcal{Q}_{g}}(\widetilde{\mathcal{S}})\right|_{\mathcal{Q}_{l}+\mathcal{Q}_{g}=\mathcal{Q}_{0}, \alpha_{k}, m_{k}, \mathcal{E}_{k}} & =\frac{U_{l}}{T_{l}}-\frac{U_{g}}{T_{g}} \\
\left.\partial_{\mathcal{E}_{g}}(\widetilde{\mathcal{S}})\right|_{\mathcal{E}_{l}+\mathcal{E}_{g}=\mathcal{E}_{0}, \alpha_{k}, m_{k}, \mathcal{Q}_{k}} & =\frac{1}{T_{g}}-\frac{1}{T_{l}} .
\end{aligned}
$$

Equation (5.4) can thus be written

$$
\begin{aligned}
\partial_{t}(\widetilde{\mathcal{S}})+\partial_{x}\left(\frac{\mathcal{Q}_{l}}{m_{l}} \mathcal{S}_{l}+\frac{\mathcal{Q}_{g}}{m_{g}} \mathcal{S}_{g}\right)= & \left.\partial_{\alpha_{g}}(\widetilde{\mathcal{S}})\right|_{\alpha_{l}+\alpha_{g}=1, m_{k}, \mathcal{Q}_{k}, \mathcal{E}_{k}} S_{1, g}^{\prime}+\left.\partial_{m_{g}}(\widetilde{\mathcal{S}})\right|_{m_{l}+m_{g}=m_{0}, \alpha_{k}, \mathcal{Q}_{k}, \mathcal{E}_{k}} S_{2, g}^{\prime} \\
& +\left.\partial_{\mathcal{Q}_{g}}(\widetilde{\mathcal{S}})\right|_{\mathcal{Q}_{l}+\mathcal{Q}_{g}=\mathcal{Q}_{0}, \alpha_{k}, m_{k}, \mathcal{E}_{k}} S_{3, g}^{\prime}+\left.\partial_{\mathcal{E}_{g}}(\widetilde{\mathcal{S}})\right|_{\mathcal{E}_{l}+\mathcal{E}_{g}=\mathcal{E}_{0}, \alpha_{k}, m_{k}, \mathcal{Q}_{k}} S_{4, g}^{\prime} .
\end{aligned}
$$

This form of equation (5.9) is the key point in the definition of the source terms proposed in the following. Indeed, the concavity of the entropy can be used to propose source terms that are non-classical for a two-fluid two-velocity approach, but that are classical for homogeneous one-velocity models.

\subsection{First set of closure laws}

Thanks to the form of equation (5.9), a first form for the source terms $S_{k, g}^{\prime}, k=1 . .4$ is proposed:

$$
S_{1, g}^{\prime}=\frac{\overline{\alpha_{g}}-\alpha_{g}}{\Lambda\left(W_{g l}\right)}, S_{2, g}^{\prime}=\frac{\overline{m_{g}}-m_{g}}{\Lambda\left(W_{g l}\right)}, S_{3, g}^{\prime}=\frac{\overline{\mathcal{Q}_{g}}-\mathcal{Q}_{g}}{\Lambda\left(W_{g l}\right)}, S_{4, g}^{\prime}=\frac{\overline{\mathcal{E}_{g}}-\mathcal{E}_{g}}{\Lambda\left(W_{g l}\right)} ;
$$

where $\left(W_{g l}\right) \mapsto \Lambda\left(W_{g l}\right)$ is a positive function that represents a time-scale; and where the quantities $\overline{W_{g}}=$ $\left(\overline{\alpha_{g}}, \overline{m_{g}}, \overline{\mathcal{Q}_{g}}, \overline{\mathcal{E}_{g}}\right)$ still have to be defined. With these choices, equation (5.9) reads:

$$
\partial_{t}(\widetilde{\mathcal{S}})+\partial_{x}\left(\frac{\mathcal{Q}_{l}}{m_{l}} \mathcal{S}_{l}+\frac{\mathcal{Q}_{g}}{m_{g}} \mathcal{S}_{g}\right)=\frac{1}{\Lambda\left(W_{g l}\right)} \nabla_{W_{g}} \widetilde{\mathcal{S}}_{g, 0} \cdot\left(\overline{W_{g}}-W_{g}\right)
$$

where the entropy $\widetilde{\mathcal{S}}_{g, 0}$ is introduced on the basis of the mixture entropy $\widetilde{\mathcal{S}}$. It is defined on ] $0,1[\times] 0, m_{0}[\times \mathbb{R} \times] 0, \mathcal{E}_{0}\left[\subset \mathcal{H}_{s, g}\right.$, with $m_{0}=m_{l}+m_{g}$ and $\mathcal{E}_{0}=\mathcal{E}_{l}+\mathcal{E}_{g}$ by:

$$
\widetilde{\mathcal{S}}_{g, 0}:\left(\begin{array}{l}
\left.W_{g} \in\right] 0,1[\times] 0, m_{0}[\times \mathbb{R} \times] 0, \mathcal{E}_{0}[\rightarrow \mathbb{R} \\
W_{g} \mapsto \widetilde{\mathcal{S}}\left(W_{g}, W_{0}-W_{g}\right)
\end{array}\right)
$$

In definition (5.12), the liquid variable $W_{l}$ is deduced from the gas variables $W_{g}$ by a conservation relation $W_{l}+W_{g}=W_{0}=\left(1, m_{0}, \mathcal{Q}_{0}, \mathcal{E}_{0}\right)$. Hence $\left(W_{g}, W_{0}-W_{g}\right)$ belongs to $\widetilde{H}_{s, 0}$. It can easily be shown that $\widetilde{\mathcal{S}}_{g, 0}$ inherits the concavity property from $\widetilde{\mathcal{S}}$.

Proposition 5.1. The entropy $W_{g} \mapsto \widetilde{\mathcal{S}}_{g, 0}\left(W_{g}\right)$ defined by $(5.12)$ is concave on $] 0,1[\times] 0, m_{0}[\times \mathbb{R} \times] 0, \mathcal{E}_{0}[$.

Proof. Let us choose $W_{g}$ and $W_{g}^{\prime}$ in $] 0,1[\times] 0, m_{0}[\times \mathbb{R} \times] 0, \mathcal{E}_{0}[$, and let $a$ be in $[0,1]$. We then get:

$$
\begin{aligned}
\widetilde{\mathcal{S}}_{g, 0}\left(a W_{g}+(1-a) W_{g}^{\prime}\right) & =\widetilde{\mathcal{S}}\left(a W_{g}+(1-a) W_{g}^{\prime}, W_{0}-a W_{g}-(1-a) W_{g}^{\prime}\right) \\
& =\widetilde{\mathcal{S}}\left(a W_{g}+(1-a) W_{g}^{\prime}, a\left(W_{0}-W_{g}\right)-(1-a)\left(W_{0}-W_{g}^{\prime}\right)\right) .
\end{aligned}
$$

It immediately arises from the concavity property of $\widetilde{\mathcal{S}}$ that:

$$
\widetilde{\mathcal{S}}_{g, 0}\left(a W_{g}+(1-a) W_{g}^{\prime}\right) \leq a \widetilde{\mathcal{S}}\left(W_{g}, W_{0}-W_{g}\right)+(1-a) \widetilde{\mathcal{S}}\left(W_{g}^{\prime}, W_{0}-W_{g}^{\prime}\right)=a \widetilde{\mathcal{S}}_{g, 0}\left(W_{g}\right)+(1-a) \widetilde{\mathcal{S}}_{g, 0}\left(W_{g}^{\prime}\right) ;
$$

and thus that $\widetilde{\mathcal{S}}_{g, 0}$ is concave on $] 0,1[\times] 0, m_{0}[\times \mathbb{R} \times] 0, \mathcal{E}_{0}[$. 
Thanks to Proposition 5.1, we obtain from (5.11) that

$$
\partial_{t}(\widetilde{\mathcal{S}})+\partial_{x}\left(\frac{\mathcal{Q}_{l}}{m_{l}} \mathcal{S}_{l}+\frac{\mathcal{Q}_{g}}{m_{g}} \mathcal{S}_{g}\right) \geq \frac{\widetilde{\mathcal{S}}_{g, 0}\left(\overline{W_{g}}\right)-\widetilde{\mathcal{S}}_{g, 0}\left(W_{g}\right)}{\Lambda\left(W_{g l}\right)}
$$

Hence, in order to ensure the growth of the entropy $\widetilde{\mathcal{S}}$ associated to the entropy-flux $\left(\mathcal{Q}_{l} / m_{l} \mathcal{S}_{l}+\mathcal{Q}_{g} / m_{g} \mathcal{S}_{g}\right)$, it could be sufficient to choose an equilibrium state $\left.\overline{W_{g}} \in\right] 0,1[\times] 0, m_{0}[\times \mathbb{R} \times] 0, \mathcal{E}_{0}[$ such that:

$$
\left.\forall W_{g} \in\right] 0,1[\times] 0, m_{0}[\times \mathbb{R} \times] 0, \mathcal{E}_{0}\left[, \quad \widetilde{\mathcal{S}}_{g, 0}\left(\overline{W_{g}}\right) \geq \widetilde{\mathcal{S}}_{g, 0}\left(W_{g}\right) .\right.
$$

Unfortunately, if the entropy $W_{g} \mapsto \widetilde{\mathcal{S}}_{g, 0}\left(W_{g}\right)$ is concave with respect to $W_{g}$, the momentum component of $W_{g}$ can not be signed and the domain of definition $] 0,1[\times] 0, m_{0}[\times \mathbb{R} \times] 0, \mathcal{E}_{0}\left[\subset \mathcal{H}_{s, g}\right.$ of $W_{g} \mapsto \widetilde{\mathcal{S}}_{g, 0}\left(W_{g}\right)$ is thus not bounded. As a consequence, the state $\overline{W_{g}}$ may itself be not bounded because of the momentum component. An other treatment of the momentum is then proposed in the next section.

\subsection{Second set of closure laws}

We propose here to treat the momentum component $\mathcal{Q}_{g}$ of the variable $W_{g}$ differently in order to work with an entropy whose domain of definition is bounded. For this purpose we define the variable $Y_{g}=\left(\alpha_{g}, m_{g}, \mathcal{E}_{g}\right) \in$ ] $0,1[\times] 0, m_{0}[\times] 0, \mathcal{E}_{0}\left[\right.$, with $m_{0}=m_{l}+m_{g}$ and $\mathcal{E}_{0}=\mathcal{E}_{l}+\mathcal{E}_{g}$, and we consider the following source terms:

$$
S_{1, g}^{\prime}=\frac{\overline{\alpha_{g}}-\alpha_{g}}{\Lambda\left(W_{g l}\right)}, \quad S_{2, g}^{\prime}=\frac{\overline{m_{g}}-m_{g}}{\Lambda\left(W_{g l}\right)}, \quad S_{3, g}^{\prime}=K_{3}^{\prime}\left(W_{g l}\right)\left(\frac{U_{l}}{T_{l}}-\frac{U_{g}}{T_{g}}\right), \quad S_{4, g}^{\prime}=\frac{\overline{\mathcal{E}_{g}}-\mathcal{E}_{g}}{\Lambda\left(W_{g l}\right)},
$$

where $K_{3}^{\prime}\left(W_{g l}\right)$ is a positive function. Equation 5.9 then becomes:

$$
\partial_{t}(\widetilde{\mathcal{S}})+\partial_{x}\left(\frac{\mathcal{Q}_{l}}{m_{l}} \mathcal{S}_{l}+\frac{\mathcal{Q}_{g}}{m_{g}} \mathcal{S}_{g}\right)=\frac{1}{\Lambda\left(W_{g l}\right)} \nabla_{Y_{g}} \widetilde{\mathcal{S}}_{g, Y, 0} \cdot\left(\overline{Y_{g}}-Y g\right)+K_{3}^{\prime}\left(W_{g l}\right)\left(\frac{U_{g}}{T_{g}}-\frac{U_{l}}{T_{l}}\right)^{2}
$$

where the entropy $\widetilde{\mathcal{S}}_{g, Y, 0}$ is defined for $\left.Y_{g}=\left(\alpha_{g}, m_{g}, \mathcal{E}_{g}\right) \in\right] 0,1[\times] 0, m_{0}[\times] 0, \mathcal{E}_{0}[$ for fixed phasic momentums:

$$
\widetilde{\mathcal{S}}_{g, Y, 0}:\left(\begin{array}{l}
\left.Y_{g} \in\right] 0,1[\times] 0, m_{0}[\times] 0, \mathcal{E}_{0}[\rightarrow \mathbb{R} \\
W_{g}=\left(\alpha_{g}, m_{g}, \mathcal{Q}_{g, 0}, \mathcal{E}_{g}\right) \mapsto \widetilde{\mathcal{S}}\left(W_{g}, W_{0}-W_{g}\right)
\end{array}\right)
$$

where $W_{0}=\left(1, m_{0}, \mathcal{Q}_{g, 0}+\mathcal{Q}_{l, 0}, \mathcal{E}_{0}\right)$. In the definition of $\widetilde{\mathcal{S}}_{g, Y, 0}$, the momentums $\mathcal{Q}_{k, 0}$ can take any bounded value since only the derivatives with respect to $Y_{g}$ play a role. The entropy $\widetilde{\mathcal{S}}_{g, Y, 0}$ inherits from the entropy $\widetilde{\mathcal{S}}_{g, 0}$ the following property.

Proposition 5.2. The entropy $Y_{g} \mapsto \widetilde{\mathcal{S}}_{g, Y, 0}\left(Y_{g}\right)$ defined by $(5.17)$ is concave on $] 0,1[\times] 0, m_{0}[\times] 0, \mathcal{E}_{0}[$.

Proof. The proof mimics the proof of Proposition 5.1.

Using the concavity of $Y_{g} \mapsto \widetilde{\mathcal{S}}_{g, Y, 0}\left(Y_{g}\right)$, on can obtain the following inequality from equation (5.16):

$$
\partial_{t}(\widetilde{\mathcal{S}})+\partial_{x}\left(\frac{\mathcal{Q}_{l}}{m_{l}} \mathcal{S}_{l}+\frac{\mathcal{Q}_{g}}{m_{g}} \mathcal{S}_{g}\right) \geq \frac{\widetilde{\mathcal{S}}_{g, Y, 0}\left(\overline{Y_{g}}\right)-\widetilde{\mathcal{S}}_{g, Y, 0}\left(Y_{g}\right)}{\Lambda\left(W_{g l}\right)}+\frac{1}{\Lambda_{u}\left(W_{g l}\right)}\left(\frac{U_{g}}{T_{g}}-\frac{U_{l}}{T_{l}}\right)^{2}
$$

On the contrary to the entropy $\widetilde{\mathcal{S}}_{g, 0}$, the entropy $\widetilde{\mathcal{S}}_{g, Y, 0}$ is concave on a bounded domain. Hence, if we exclude the single-phase flow situations, there exists at least one equilibrium state $\overline{Y_{g}} \in[0,1] \times\left[0, m_{0}\right] \times\left[0, \mathcal{E}_{0}\right]$ such that

$$
\forall Y_{g} \in[0,1] \times\left[0, m_{0}\right] \times\left[0, \mathcal{E}_{0}\right], \quad \widetilde{\mathcal{S}}_{g, Y, 0}\left(\overline{Y_{g}}\right) \geq \widetilde{\mathcal{S}}_{g, Y, 0}\left(Y_{g}\right)
$$


We can thus conclude that the choice of source terms (5.15) leads to an entropy inequality for the mixture entropy $\widetilde{\mathcal{S}}$ when associated with the entropy-flux $\left(\mathcal{Q}_{l} / m_{l} \mathcal{S}_{l}+\mathcal{Q}_{g} / m_{g} \mathcal{S}_{g}\right)$ :

$$
\partial_{t}(\widetilde{\mathcal{S}})+\partial_{x}\left(\frac{\mathcal{Q}_{l}}{m_{l}} \mathcal{S}_{l}+\frac{\mathcal{Q}_{g}}{m_{g}} \mathcal{S}_{g}\right) \geq 0
$$

Since $\widetilde{\mathcal{S}}_{g, Y, 0}$ is not strictly concave, the equilibrium state $\overline{Y_{g}}$ may be non-unique, but it exists and it is defined as a state such that:

$$
\widetilde{\mathcal{S}}_{g, Y, 0}\left(\overline{Y_{g}}\right)=\max _{Y_{g} \in[0,1] \times\left[0, m_{0}\right] \times\left[0, \mathcal{E}_{0}\right], \mathcal{Q}_{g}=\mathcal{Q}_{g, 0}, \mathcal{Q}_{l}=\mathcal{Q}_{l, 0}}\left(\widetilde{\mathcal{S}}_{g, Y, 0}\left(Y_{g}\right)\right) .
$$

Nevertheless, this proposition (5.15) leads to a drag force $S_{3, g}^{\prime}$ that is more than a mechanical effect. Indeed, the classical form for the drag force as introduced in Section 4 by Definition 4.4 tends to decrease the relative velocity independently of the thermodynamical quantities. This is not the case with the source terms $S_{3, g}^{\prime}$ as proposed above. This is due to the fact that the source terms (5.15) have not been split into elementary contributions, as in Section 4. In fact we have:

$$
\left(\frac{U_{g}}{T_{g}}-\frac{U_{l}}{T_{l}}\right)=\frac{1}{T_{g}}\left(U_{g}-U_{l}\right)+U_{l}\left(\frac{1}{T_{l}}-\frac{1}{T_{g}}\right)
$$

In the third proposition, we introduce a splitting of the source terms that mimics the splitting presented in Section 4.

\subsection{Third set of closure laws}

We modify $S_{3, g}^{\prime}$ and $S_{4, g}^{\prime}$ while keeping $S_{1, g}^{\prime}$ and $S_{2, g}^{\prime}$ unchanged. These new choices mimic the splitting of the source terms $S_{3, g}$ and $S_{4, g}$ defined in Section 4 . We then introduce two velocities $\widetilde{V}^{\prime}$ and an energy $\widetilde{E}^{\prime}$, that will be defined in the following. Moreover, the momentums are not treated together with the thermodynamical variables. We thus focus in this section on the entropy $\eta$ and not on the entropy $\mathcal{S}$ as in the two previous sections. The entropy $\widetilde{\eta}_{g, 0}$ is then defined for the variable $Z_{g}=\left(\alpha_{g}, m_{g}, \varepsilon_{g}\right)$ on the basis of the mixture entropy $\eta$ defined in Section 3.1 by definition (3.1):

$$
\widetilde{\eta}_{g, 0}:\left(\begin{array}{l}
\left.Z_{g} \in\right] 0,1[\times] 0, m_{0}[\times] 0, \varepsilon_{0}[\rightarrow \mathbb{R} \\
Z_{g} \mapsto \widetilde{\eta}\left(Z_{g}, Z_{0}-Z_{g}\right)
\end{array}\right),
$$

with $Z_{0}=\left(\alpha_{0}, m_{0}, \varepsilon_{0}\right)=\left(1, m_{l}+m_{g}, \varepsilon_{l}+\varepsilon_{g}\right)$. We can then state the following property.

Proposition 5.3. The entropy $Z_{g} \mapsto \widetilde{\eta}_{g, 0}\left(Z_{g}\right)$ defined by $(5.22)$ is concave on $] 0,1[\times] 0, m_{0}[\times] 0, \varepsilon_{0}[$ and it possesses a unique maximum $\overline{Z_{g}}=\left(\overline{\alpha_{g}}, \overline{m_{g}}, \overline{\varepsilon_{g}}\right)$ on $] 0,1[\times] 0, m_{0}[\times] 0, \varepsilon_{0}[$.

Proof. The proof of the concavity of $\widetilde{\eta}_{g, 0}$ is based on Theorem 3.3 and its proof mimics the proof of Proposition 5.1. The uniqueness of the maximum of $\widetilde{\eta}_{g, 0}$ is inherited from the Corollary 3.4 of the entropy $\widetilde{\eta}$. Obviously, as for Corollary 3.4, we exclude the single-phase flow situations.

We introduce now the following splitting of the source terms:

$$
\left\{\begin{array}{l}
S_{1, g}^{\prime}=\frac{\overline{\alpha_{g}}-\alpha_{g}}{\Lambda\left(W_{g l}\right)}, \\
S_{2, g}^{\prime}=\frac{\overline{m_{g}}-m_{g}}{\Lambda\left(W_{g l}\right)} \\
S_{3, g}^{\prime}=D_{u, g}+\widetilde{V}^{\prime} S_{2, g}^{\prime}, \\
S_{4, g}^{\prime}=\frac{\overline{\varepsilon_{g}}-\varepsilon_{g}}{\Lambda\left(W_{g l}\right)}+\bar{V} D_{u, g}+\widetilde{E}^{\prime} S_{2, g}^{\prime},
\end{array}\right.
$$


where the drag force $D_{u, g}$ and the velocity $\bar{V}$ have been defined in Section 4 (through Def. 4.4). The equilibrium state $\overline{Z_{g}}=\left(\overline{\alpha_{g}}, \overline{m_{g}}, \overline{\varepsilon_{g}}\right)$ corresponds to the maximum of the entropy $\widetilde{\eta}_{g, 0}$ on $] 0,1[\times] 0, m_{0}[\times] 0, \varepsilon_{0}[$. Its is unique and it is defined as a state such that:

$$
\widetilde{\eta}_{g, 0}\left(\overline{Z_{g}}\right)=\max _{\left.Z_{g} \in\right] 0,1[\times] 0, m_{0}[\times] 0, \varepsilon_{0}[}\left(\widetilde{\eta}_{g, 0}\left(Z_{g}\right)\right) .
$$

The function $W_{g l} \mapsto \Lambda\left(W_{g l}\right)$ must be positive. When using relations (A.1)-(A.4), equation (5.4) can be written:

$$
\begin{aligned}
\partial_{t}(\widetilde{\mathcal{S}})+\partial_{x}\left(\frac{\mathcal{Q}_{l}}{m_{l}} \mathcal{S}_{l}+\frac{\mathcal{Q}_{g}}{m_{g}} \mathcal{S}_{g}\right)= & \frac{1}{\Lambda\left(W_{g l}\right)} \nabla_{Z_{g}} \widetilde{\eta}_{g, 0} \cdot\left(\overline{Z_{g}}-Z g\right)+\left(\frac{U_{l}}{T_{l}}-\frac{U_{g}}{T_{g}}+\bar{V}\left(\frac{1}{T_{g}}-\frac{1}{T_{l}}\right)\right) D_{u, g} \\
& +\left(\frac{U_{g}^{2} / 2}{T_{g}}-\frac{U_{l}^{2} / 2}{T_{l}}+\widetilde{V}^{\prime}\left(\frac{U_{l}}{T_{l}}-\frac{U_{g}}{T_{g}}\right)+\widetilde{E}^{\prime}\left(\frac{1}{T_{g}}-\frac{1}{T_{l}}\right)\right) S_{2, g}^{\prime} .
\end{aligned}
$$

In order to get a entropy inequality, the three terms on the right hand side of (5.25) have to be positive. The first terms is indeed positive since $\widetilde{\eta}_{g, 0}$ is concave and thanks to the definition (5.24) of $\overline{Z_{g}}$. For the second and third terms, we adopt the same choices than for the classical closures of Section 4: $\widetilde{V}^{\prime}=\bar{V}=\left(U_{l}+U_{g}\right) / 2$ and $\widetilde{E}^{\prime}=U_{l} U_{g} / 2$. The third term of the right hand side of the entropy equation (5.25) then vanishes, and we get:

$$
\partial_{t}(\widetilde{\mathcal{S}})+\partial_{x}\left(\frac{\mathcal{Q}_{l}}{m_{l}} \mathcal{S}_{l}+\frac{\mathcal{Q}_{g}}{m_{g}} \mathcal{S}_{g}\right)=\frac{1}{\Lambda\left(W_{g l}\right)} \nabla_{Z_{g}} \widetilde{\eta}_{g, 0} \cdot\left(\overline{Z_{g}}-Z g\right)+\left(\frac{1}{2 T_{l}}+\frac{1}{2 T_{g}}\right)\left(U_{l}-U_{g}\right) D_{u, g}
$$

As for the drag force term of Section 4, we choose:

$$
D_{u, g}=K_{3}\left(W_{g l}\right)\left(\frac{1}{2 T_{g}}+\frac{1}{2 T_{l}}\right)\left(U_{l}-U_{g}\right)=K_{3, u}\left(W_{g l}\right)\left(U_{l}-U_{g}\right),
$$

where $W_{g l} \mapsto K_{3, u}\left(W_{g l}\right)$ is a positive function. With all these choices, we recover the entropy inequality (5.20) for the mixture entropy $\widetilde{\mathcal{S}}$ when associated with the entropy-flux $\left(\mathcal{Q}_{l} / m_{l} \mathcal{S}_{l}+\mathcal{Q}_{g} / m_{g} \mathcal{S}_{g}\right)$.

The sources terms proposed in this section involve two parameters, whereas the classical closures involve four parameters. The first parameter is the parameter $K_{3, u}$ which rules the kinematic equilibrium, and which is the same than in Section 4. Concerning the return to the thermodynamical equilibrium, the source terms of the present section only involve one time-scale $\Lambda$ whereas the classical source terms are ruled by three independent parameters. But the great advantage of the present source terms is that they are far more easy to integrate when dealing with numerical simulations. Indeed, using a fractional step approach for the discretization of the whole model, the thermodynamical relaxation towards the equilibrium can be done using simple and robust schemes, see $[19,20]$ for instance.

\subsection{Fourth set of closure laws}

We focus now on the entropy $m_{k} \mapsto \eta_{m, k}\left(m_{k}\right)$ introduced in Section 3.1. On the contrary to the three previous sections, we use here system (2.1) as for the classical source terms of Section 4. Thanks to Proposition 3.7, we know that $m_{k} \mapsto \eta_{m, k}\left(m_{k}\right)$ is strictly concave. We then define the mixture entropy $\eta_{m, 0}$ as:

$$
\eta_{m, 0}:\left(\begin{array}{l}
\left.m_{g} \in\right] 0, m_{0}[\rightarrow \mathbb{R} \\
m_{g} \mapsto \eta_{m, l}\left(m_{0}-m_{g}\right)+\eta_{m, g}\left(m_{g}\right)
\end{array}\right) .
$$

For the entropy $\eta_{m, 0}$, the fractions and the energies are fixed quantities. Then, Proposition 3.2 leads to the following property for the mixture entropy $m_{g} \mapsto \eta_{m, 0}\left(m_{g}\right)$.

Proposition 5.4. The entropy $m_{g} \mapsto \eta_{m, 0}\left(m_{g}\right)$ belongs to $C^{2}(] 0, m_{0}[, \mathbb{R})$ and it is strictly concave on $] 0, m_{0}[$. 
Proof. The entropy $m_{g} \mapsto \eta_{m, 0}$ directly inherits its regularity property from the phasic entropies $\eta_{m, k}$ (see Prop. 3.2). In order to prove the concavity results, formula (A.11) and (A.14) from appendix A are used. We then get the first derivative $m_{g} \mapsto \eta_{m, 0}$ :

$$
\eta_{m, 0}^{\prime}\left(m_{g}\right)=\eta_{m, g}^{\prime}\left(m_{g}\right)-\eta_{m, l}^{\prime}\left(m_{0}-m_{g}\right)=\frac{\mu_{l}}{T_{l}}-\frac{\mu_{g}}{T_{g}},
$$

and the second derivative of $m_{g} \mapsto \eta_{m, 0}$;

$$
\eta_{m, 0}^{\prime \prime}\left(m_{g}\right)=\frac{1}{m_{g}}\left(\frac{\alpha_{g}^{2}}{m_{g}^{2}}, \frac{\varepsilon_{g}^{2}}{m_{g}^{2}}\right) \cdot s_{g}^{\prime \prime} \cdot\left(\begin{array}{c}
\frac{\alpha_{g}^{2}}{m_{g}^{2}} \\
\frac{\varepsilon_{g}^{2}}{m_{g}^{2}}
\end{array}\right)+\frac{1}{m_{0}-m_{g}}\left(\frac{\alpha_{l}^{2}}{\left(m_{0}-m g\right)^{2}}, \frac{\varepsilon_{l}^{2}}{\left(m_{0}-m_{g}\right)^{2}}\right) \cdot s_{l}^{\prime \prime} \cdot\left(\begin{array}{c}
\frac{\alpha_{l}^{2}}{\left(m_{0}-m_{g}\right)^{2}} \\
\frac{\varepsilon_{l}^{2}}{\left(m_{0}-m_{g}\right)^{2}}
\end{array}\right) .
$$

Since $\left.m_{g} \in\right] 0, m_{0}\left[\right.$ and since the phasic entropies $s_{k}$ are strictly concave, we finally get that,

$$
\left.\forall m_{g} \in\right] 0, m_{0}\left[, \quad \eta_{m, 0}^{\prime \prime}\left(m_{g}\right)<0\right.
$$

which prove that $m_{g} \mapsto \eta_{m, 0}$ is strictly concave on $] 0, m_{0}[$. This ends the proof of Proposition 5.4.

In order to take advantage of this property, we define the source terms:

$$
\left\{\begin{array}{l}
S_{1, g}=K_{1}\left(W_{g l}\right)\left(P_{g}-P_{l}\right), \\
S_{2, g}=\frac{\overline{m_{g}}-m_{g}}{\Lambda_{m}\left(W_{g l}\right)}, \widetilde{V} S_{2, g}^{\prime}, \\
S_{3, g}=D_{u, g}+\bar{H} D_{u, g}+\bar{H} S_{2, g}^{\prime}, \\
S_{4, g}=\Psi_{g}+\bar{V}
\end{array}\right.
$$

where $W_{g l} \mapsto \Lambda_{m}\left(W_{g l}\right)$ is a positive function. For these source terms, only the mass transfer term $S_{2, g}$ differs from the classical source terms of Section 4 . The equilibrium mass $\overline{m_{g}}$ involved in the mass transfer term $S_{2, g}$ is defined as the point that maximizes the entropy $\eta_{m, 0}$, for given fractions $\alpha_{k}$ and for given internal energies $\varepsilon_{k}$ :

$$
\eta_{m, 0}\left(\overline{m_{g}}\right)=\max _{\left.m_{g} \in\right] 0, m_{0}[}\left(\eta_{m, 0}\left(m_{g}\right)\right) .
$$

As in previous sections, we assume that single-phase flows are not reached. Therefore the maximum for $\eta_{m, 0}$ can not be reached for $m_{g} \rightarrow 0^{+}$or $m_{g} \rightarrow m_{0}^{-}$. If we still retain the closures $\widetilde{V}=\bar{V}=\left(U_{l}+U_{g}\right) / 2$ and $\bar{H}=U_{l} U_{g} / 2$, the entropy equation (4.2) then becomes:

$$
\begin{aligned}
\partial_{t}(\widetilde{\mathcal{S}})+\partial_{x}\left(\frac{\mathcal{Q}_{l}}{m_{l}} \mathcal{S}_{l}+\frac{\mathcal{Q}_{g}}{m_{g}} \mathcal{S}_{g}\right)= & \frac{\left(P_{g}-P_{l}\right)}{(1-\beta) T_{g}+\beta T_{l}} S_{1, g}+\left(\frac{\mu_{l}}{T_{l}}-\frac{\mu_{g}}{T_{g}}\right) S_{2, g} \\
& +\left(\frac{1}{2 T_{l}}+\frac{1}{2 T_{g}}\right)\left(U_{l}-U_{g}\right) D_{u, g}+\left(\frac{1}{T_{g}}-\frac{1}{T_{l}}\right) \Psi_{g}
\end{aligned}
$$

If the derivative of $\eta_{m, 0}$ given by (5.28) is introduced in (5.32), we get:

$$
\begin{aligned}
\partial_{t}(\widetilde{\mathcal{S}})+\partial_{x}\left(\frac{\mathcal{Q}_{l}}{m_{l}} \mathcal{S}_{l}+\frac{\mathcal{Q}_{g}}{m_{g}} \mathcal{S}_{g}\right)= & \frac{1}{\Lambda_{m}\left(W_{g l}\right)} \nabla_{m_{g}} \eta_{m, 0} \cdot\left(\overline{m_{g}}-m g\right)+\frac{\left(P_{g}-P_{l}\right)}{(1-\beta) T_{g}+\beta T_{l}} S_{1, g} \\
& +\left(\frac{1}{2 T_{l}}+\frac{1}{2 T_{g}}\right)\left(U_{l}-U_{g}\right) D_{u, g}+\left(\frac{1}{T_{g}}-\frac{1}{T_{l}}\right) \Psi_{g}
\end{aligned}
$$

The first term of he right hand side of (5.33) is non-negative thanks to the concavity of $\eta_{m, 0}$ and thanks to the definition of $\overline{m_{g}}$. Therefore, in order to get an entropy inequality from (5.33), we can then choose $\Psi_{g}$ and $D_{u, g}$ as in Section 4:

$$
D_{u, g}=K_{3, u}\left(W_{g l}\right)\left(U_{l}-U_{g}\right), \quad \text { and } \quad \Psi_{g}=K_{4}\left(W_{g l}\right)\left(\frac{1}{T_{g}}-\frac{1}{T_{l}}\right) .
$$


We recover the entropy inequality (5.20) for the mixture entropy $\widetilde{\mathcal{S}}$ when associated with the entropy-flux $\left(\mathcal{Q}_{l} / m_{l} \mathcal{S}_{l}+\mathcal{Q}_{g} / m_{g} \mathcal{S}_{g}\right)$.

As in the previous section, this fourth set of closure laws presents an advantage for the numerical simulation because it allows to simplify the numerical integration of the mass transfer using fractional step approach. But, on the contrary to the source terms of the previous section, we keep here four parameters for the definition of relaxation paths: $K_{1}\left(W_{g l}\right), K_{3, u}\left(W_{g l}\right), K_{4}\left(W_{g l}\right)$ and $\Lambda_{m}\left(W_{g l}\right)$.

Remark 5.5. The same idea can be applied to system (5.1), and it would lead to a different source term for the fraction:

$$
S_{1, g}=K_{1}\left(W_{g l}\right)\left(P_{g} / T_{g}-P_{l} / T_{l}\right)
$$

nevertheless, in this fourth set of closure laws we intend to keep a formulation close to the classical source terms of Section 4, that is why we have preferred system (2.1).

\section{CONCLusion}

Four non-classical sets of source terms have been proposed for the two-fluid two-pressure Baer-Nunziato type model. It should be quoted that the approach proposed in the sequel is not restricted to two-velocity models. It can also be applied to a wide range of multi-fluid multi-velocity models, and even to homogeneous models.

The set of closure laws of Section 5.3 is very similar to the form of the source terms of the homogeneous models. From a numerical point of view, efficient numerical schemes for instance based on those proposed in $[19,20]$ can thus be used. This kind of source terms and the associated numerical schemes have several advantages:

- the pressure, temperature and chemical potential relaxation effects are solved simultaneously, whatever the stiffness of the relaxation effects may be;

- the numerical integration relies on an almost exact integration, which provides a good accuracy and robustness of the scheme;

- there is no restriction on the complexity of the form of the EOS used for each phase;

- this scheme is rather efficient in terms of CPU-time because it only requires to solve one non-linear equation (to compute the equilibrium state).

However, when turning to the physical point of view, the pressure, temperature and chemical potential relaxations are driven by a unique time-scale, which may sometimes be too restrictive. The set of closure laws of Section 5.4 overtakes this limitation, since each relaxation effect is associated with its own time-scale. Nevertheless, this choice also presents drawbacks:

- in order to discretize the pressure, temperature and chemical potential relaxation in a coupled manner a complex numerical scheme is required;

- moreover, if the three relaxation effects are solved separately, each one is associated with at least one computation of the solution of a non-linear equation, which may be CPU-consuming for complex EOS.

Hence, the set of closure laws of Sections 5.3 and 5.4 have both drawbacks and advantages. The choice between these two sets of source terms should be driven by the physical configuration to be simulated and by the informations that have to be collected with the numerical simulations.

\section{Appendix A. Derivative terms of the miXture entropy $\mathcal{S}$}

We report here the derivatives of the entropy $W_{g l} \mapsto \mathcal{S}\left(W_{g l}\right)$ defined in Section 3 by (3.34). The first derivatives of $\mathcal{S}$ are obviously the basis of the computation of the second derivatives, but they also play an important role in the definition of admissible source terms in Section 5. In the following, for the sake of readability 
$\eta_{k}\left(\alpha_{k}, m_{k}, \mathcal{E}_{k}-\frac{Q_{k}^{2}}{2 m_{k}}\right)$ will be replaced by $\eta_{k}$ (we omit the arguments). The first derivative terms for the phasic entropies $\mathcal{S}_{k}$ read:

$$
\begin{aligned}
& \partial_{\alpha_{k}}\left(\mathcal{S}_{k}\right)_{\mid m_{k}, \mathcal{E}_{k}, Q_{k}}\left(W_{k}\right)=\partial_{\alpha_{k}}\left(\eta_{k}\right)_{\mid m_{k}, \varepsilon_{k}} \\
& \partial_{m_{k}}\left(\mathcal{S}_{k}\right)_{\mid \alpha_{k}, \mathcal{E}_{k}, Q_{k}}\left(W_{k}\right)=\partial_{m_{k}}\left(\eta_{k}\right)_{\mid \alpha_{k}, \varepsilon_{k}}+\frac{Q_{k}^{2}}{2 m_{k}^{2}} \partial_{\mathcal{\varepsilon}_{k}}\left(\eta_{k}\right)_{\mid \alpha_{k}, m_{k}} \\
& \partial_{\mathcal{E}_{k}}\left(\mathcal{S}_{k}\right)_{\mid \alpha_{k}, m_{k}, Q_{k}}\left(W_{k}\right)=\partial_{\varepsilon_{k}}\left(\eta_{k}\right)_{\mid \alpha_{k}, m_{k}} \\
& \partial_{Q_{k}}\left(\mathcal{S}_{k}\right)_{\mid \alpha_{k}, m_{k}, \mathcal{E}_{k}}\left(W_{k}\right)=-\frac{Q_{k}}{m_{k}} \partial_{\varepsilon_{k}}\left(\eta_{k}\right)_{\mid \alpha_{k}, m_{k}} .
\end{aligned}
$$

If we introduce the first derivatives of $\eta_{k}$ with respect to $\alpha_{k}, m_{k}$ and $\varepsilon_{k}$, as expressed by equations (3.5)-(3.7), we have:

$$
\begin{aligned}
\partial_{\alpha_{k}}\left(\mathcal{S}_{k}\right)_{\mid m_{k}, \mathcal{E}_{k}, Q_{k}}\left(W_{k}\right) & =\frac{P_{k}\left(\tau_{k}, e_{k}\right)}{T_{k}\left(\tau_{k}, e_{k}\right)}, \\
\partial_{m_{k}}\left(\mathcal{S}_{k}\right)_{\mid \alpha_{k}, \mathcal{E}_{k}, Q_{k}}\left(W_{k}\right) & =\frac{\frac{Q_{k}^{2}}{2 m_{k}^{2}}-\mu_{k}\left(\tau_{k}, e_{k}\right)}{T_{k}\left(\tau_{k}, e_{k}\right)}, \\
\partial_{\mathcal{E}_{k}}\left(\mathcal{S}_{k}\right)_{\mid \alpha_{k}, m_{k}, Q_{k}}\left(W_{k}\right) & =\frac{1}{T_{k}\left(\tau_{k}, e_{k}\right)}, \\
\partial_{Q_{k}}\left(\mathcal{S}_{k}\right)_{\mid \alpha_{k}, m_{k}, \mathcal{E}_{k}}\left(W_{k}\right) & =-\frac{Q_{k}}{m_{k}} \frac{1}{T_{k}\left(\tau_{k}, e_{k}\right)},
\end{aligned}
$$

where $\tau_{k}=\alpha_{k} / m_{k}$ and $e_{k}=\mathcal{E}_{k} / m_{k}-Q_{k}^{2} /\left(2 m_{k}^{2}\right)$. It can be noticed that the entropy $W_{k} \mapsto \mathcal{S}_{k}\left(W_{k}\right)$ can thus be written:

$$
\begin{aligned}
& \mathcal{S}_{k}\left(\alpha_{k}, m_{k}, \mathcal{E}_{k}, Q_{k}\right)=\alpha_{k} \quad \partial_{\alpha_{k}}\left(\mathcal{S}_{k}\right)_{\mid m_{k}, \mathcal{E}_{k}, Q_{k}}\left(W_{k}\right)+m_{k} \quad \partial_{m_{k}}\left(\mathcal{S}_{k}\right)_{\mid \alpha_{k}, \mathcal{E}_{k}, Q_{k}}\left(W_{k}\right) \\
& +\mathcal{E}_{k} \quad \partial_{\mathcal{E}_{k}}\left(\mathcal{S}_{k}\right)_{\mid \alpha_{k}, m_{k}, Q_{k}}\left(W_{k}\right)+Q_{k} \quad \partial_{Q_{k}}\left(\mathcal{S}_{k}\right)_{\mid \alpha_{k}, m_{k}, \mathcal{E}_{k}}\left(W_{k}\right) .
\end{aligned}
$$

In order to compute the second derivatives of $\mathcal{S}_{k}$, we use equations (A.1)-(A.4) and we get:

$$
\begin{aligned}
& \partial_{\alpha_{k}, \alpha_{k}}^{2}\left(\mathcal{S}_{k}\right)\left(W_{k}\right)=\partial_{\alpha_{k}, \alpha_{k}}^{2}\left(\eta_{k}\right) \\
& \partial_{\alpha_{k}, m_{k}}^{2}\left(\mathcal{S}_{k}\right)\left(W_{k}\right)=\partial_{\alpha_{k}, m_{k}}^{2}\left(\eta_{k}\right)+\frac{Q_{k}^{2}}{2 m_{k}} \partial_{\alpha_{k}, \varepsilon_{k}}^{2}\left(\eta_{k}\right) \\
& \partial_{\alpha_{k}, \mathcal{E}_{k}}^{2}\left(\mathcal{S}_{k}\right)\left(W_{k}\right)=\partial_{\alpha_{k}, \varepsilon_{k}}^{2}\left(\eta_{k}\right) \\
& \partial_{\alpha_{k}, Q_{k}}^{2}\left(\mathcal{S}_{k}\right)\left(W_{k}\right)=-\frac{Q_{k}}{m_{k}} \partial_{\alpha_{k}, \varepsilon_{k}}^{2}\left(\eta_{k}\right) \\
& \partial_{m_{k}, m_{k}}^{2}\left(\mathcal{S}_{k}\right)\left(W_{k}\right)=-\frac{Q_{k}^{2}}{m_{k}^{3}} \partial_{\varepsilon_{k}}\left(\eta_{k}\right)_{\mid \alpha_{k}, m_{k}}+\left(1, \frac{Q_{k}^{2}}{2 m_{k}}\right) \cdot\left(\begin{array}{c}
\left.\partial_{m_{k}, m_{k}}^{2}\left(\eta_{k}\right) \partial_{m_{k}, \varepsilon_{k}}^{2}\left(\eta_{k}\right)\right) \cdot\left(\begin{array}{c}
1 \\
\partial_{m_{k}, \varepsilon_{k}}^{2}\left(\eta_{k}\right) \partial_{\varepsilon_{k}, \varepsilon_{k}}^{2}\left(\eta_{k}\right)
\end{array}\right) \\
\frac{Q_{k}^{2}}{2 m_{k}}
\end{array}\right) \\
& \partial_{m_{k}, \mathcal{E}_{k}}^{2}\left(\mathcal{S}_{k}\right)\left(W_{k}\right)=\partial_{m_{k}, \varepsilon_{k}}^{2}\left(\eta_{k}\right)+\frac{Q_{k}^{2}}{2 m_{k}} \partial_{\varepsilon_{k}, \varepsilon_{k}}^{2}\left(\eta_{k}\right) \\
& \partial_{m_{k}, Q_{k}}^{2}\left(\mathcal{S}_{k}\right)\left(W_{k}\right)=\frac{Q_{k}}{m_{k}^{2}} \partial_{\varepsilon_{k}}\left(\eta_{k}\right)_{\mid \alpha_{k}, m_{k}}-\frac{Q_{k}}{m_{k}}\left(\partial_{m_{k}, \varepsilon_{k}}^{2}\left(\eta_{k}\right)+\frac{Q_{k}^{2}}{2 m_{k}} \partial_{\varepsilon_{k}, \varepsilon_{k}}^{2}\left(\eta_{k}\right)\right) \\
& \partial_{\mathcal{E}_{k}, \mathcal{E}_{k}}^{2}\left(\mathcal{S}_{k}\right)\left(W_{k}\right)=\partial_{\varepsilon_{k}, \varepsilon_{k}}^{2}\left(\eta_{k}\right) \\
& \partial_{\mathcal{E}_{k}, Q_{k}}^{2}\left(\mathcal{S}_{k}\right)\left(W_{k}\right)=-\frac{Q_{k}}{m_{k}} \partial_{\varepsilon_{k}, \varepsilon_{k}}^{2}\left(\eta_{k}\right) \\
& \partial_{Q_{k}, Q_{k}}^{2}\left(\mathcal{S}_{k}\right)\left(W_{k}\right)=-\frac{1}{m_{k}} \partial_{\varepsilon_{k}}\left(\eta_{k}\right)_{\mid \alpha_{k}, m_{k}}+\frac{Q_{k}^{2}}{m_{k}^{2}} \partial_{\varepsilon_{k}, \varepsilon_{k}}^{2}\left(\eta_{k}\right) .
\end{aligned}
$$


Once the derivatives of the phasic entropies $\mathcal{S}_{k}$ have been written, it is easy to get the derivatives of the mixture entropy $\mathcal{S}$ thanks to the definition $(3.34)$ for $\mathcal{S}$.

\section{REFERENCES}

[1] M. Baer and J. Nunziato, A two-phase mixture theory for the deflagration-to-detonation transition (DDT) in reactive granular materials. Int. J. Multiphase Flow 12 (1986) 861-889.

[2] T. Barberon and P. Helluy, Finite volume simulation of cavitating flows. Comput. Fluids 34 (2005) 832-858.

[3] R.A. Berry, J.W. Peterson, H. Zhang, R.C. Martineau, H. Zhao, L. Zou and D. Andrs, Relap-7 theory manual. Technical report, Idaho National Laboratory, INL/EXT-14-31366 (2014).

[4] C. Chalons, A simple and accurate coupled HLL-type approximate Riemann solver for the two-fluid two-pressure model of compressible flows. Int. J. Finite Vol. (2016) https://hal.archives-ouvertes.fr/hal-01419932.

[5] F. Coquel, T. Gallouët, J.-M. Hérard and N. Seguin, Closure laws for a two-fluid two-pressure model. C. R. Math. 334 (2002) 927-932.

[6] F. Coquel, T. Gallouët, P. Helluy, J.-M. Hérard, O. Hurisse and N. Seguin, Modelling compressible multiphase flows. ESAIM: Proc. Surv. 40 (2013) 34-50.

[7] F. Coquel, J.-M. Hérard, K. Saleh and N. Seguin, Two properties of two-velocity two-pressure models for two-phase flows. Commun. Math. Sci. 12 (2014) 593-600.

[8] F. Coquel, J.-M. Hérard and K. Saleh, A positive and entropy-satisfying finite volume scheme for the baer-nunziato model. J. Comput. Phys. 330 (2017) 401-435.

[9] F. Crouzet, F. Daude, P. Galon, J.-M. Hérard, O. Hurisse and Y. Liu, Validation of a two-fluid model on unsteady liquid--vapor water flows. Comput. Fluids 119 (2015) 131-142.

[10] P. Downar-Zapolski, Z. Bilicki, L. Bolle and J. Franco, The non-equilibrium relaxation model for one-dimensional flashing liquid flow. Int. J. Multiphase Flow 22 (1996) 473-483.

[11] T. Gallouët, J.-M. Hérard and N. Seguin, Numerical modeling of two-phase flows using the two-fluid two-pressure approach. Math. Models Methods Appl. Sci. 14 (2004) 663-700.

[12] S. Gavrilyuk and R. Saurel, Mathematical and numerical modeling of two-phase compressible flows with micro-inertia. J. Comput. Phys. 175 (2002) 326-360.

[13] J. Glimm, D. Saltz and D. Sharp, Two-pressure two-phase flow. In: Advances In Nonlinear Partial Differential Equations and Related Areas: A Volume in Honor of Professor Xiaqi Ding. World Scientific (1998) 124-148.

[14] V. Guillemaud, Modelling and numerical simulation of two-phase flows using the two-fluid two-pressure approach. Theses, Université de Provence - Aix-Marseille I (2007) https://tel.archives-ouvertes.fr/tel-00169178.

[15] J.-M. Hérard and O. Hurisse, A fractional step method to compute a class of compressible gas-liquid flows. Comput. Fluids 55 (2012) 57-69.

[16] J.-M. Hérard and O. Hurisse, Computing two-fluid models of compressible water-vapour flows with mass transfer. In: 42nd AIAA Fluid Dynamics Conference and Exhibit (2012). DOI: 10.2514/6.2012-2959.

[17] J.-M. Hérard, O. Hurisse, A. Morente and K. Saleh, Application of a two-fluid model to simulate the heating of two-phase flows. In: Finite Volumes for Complex Applications VII-Elliptic, Parabolic and Hyperbolic Problems. Springer (2014) 857-864.

[18] M. Hillairet, On Baer-Nunziato multiphase flow models. ESAIM Proc. Surv. 66 (2019) 61-83.

[19] O. Hurisse, Application of an homogeneous model to simulate the heating of two-phase flows. Int. J. Finite Vol. 11 (2014) $1-37$.

[20] O. Hurisse, Numerical simulations of steady and unsteady two-phase flows using a homogeneous model. Comput. Fluids $\mathbf{1 5 2}$ (2017) 88-103.

[21] H. Jin, J. Glimm and D. Sharp, Compressible two-pressure two-phase flow models. Phys. Lett. A 353 (2006) $469-474$.

[22] Y. Liu, Contribution to the verification and the validation of an unsteady two-phase flow model. Theses, Aix-Marseille Université (2013). https://tel.archives-ouvertes.fr/tel-00864567.

[23] H. Lochon, Modelling and simulation of steam-water transients using the two-fluid approach. Theses, Aix Marseille Université (2016) https://tel.archives-ouvertes.fr/tel-01379453.

[24] H. Lochon, F. Daude, P. Galon and J.-M. Hérard, HLLC-type riemann solver with approximated two-phase contact for the computation of the baer-nunziato two-fluid model. J. Comput. Phys. 326 (2016) 733-762.

[25] S. Müller, M. Hantke and P. Richter, Closure conditions for non-equilibrium multi-component models. Continuum Mech. Thermodyn. 28 (2016) 1157-1189.

[26] D.W. Schwendeman, C.W. Wahle and A.K. Kapila, The riemann problem and a high-resolution godunov method for a model of compressible two-phase flow. J. Comput. Phys. 212 (2006) 490-526.

[27] S. Tokareva and E.F. Toro, HLLC-type Riemann solver for the Baer-Nunziato equations of compressible two-phase flow. $J$. Comput. Phys. 229 (2010) 3573-3604.

[28] N. Yanenko, Méthode à pas fractionnaires. Résolutions de problèmes polydimensionnels de physique mathématique. Armand Colin (1968). 